\title{
SZENARIEN ZUR MITTEL- UND LANGFRISTIGEN \\ ENTWICKLUNG DER ANZAHL DER \\ ERWERBSPERSONEN UND DER \\ ERWERBSTÄTIGEN IN DEUTSCHLAND
}

Axel Börsch-Supan \& Christina B. Wilke

๑ mea-Mannheim Research Institute for the Economics of Aging

L13, 17_D-68131 Mannheim_Phone +49 621 181-2773/1862_Fax +49 621 181-1863_www.mea.uni-mannheim.de 


\title{
Szenarien zur mittel- und langfristigen Entwicklung der Anzahl der Erwerbspersonen und der Erwerbstätigen in Deutschland
}

\author{
Axel Börsch-Supan und Christina B. Wilke \\ Mannheim Research Institute for the Economics of Aging (MEA)
}

\section{ZUSAMMENFASSUNG}

Dieses Papier legt Projektionen zur mittel- und langfristigen Entwicklung der Anzahl der Erwerbspersonen sowie der Erwerbstätigen in Deutschland vor.

Für unser vorsichtig optimistisches Szenario gehen wir von einer allmählichen Angleichung an die heutige Arbeitsmarktsituation in Dänemark aus. In diesem Fall ergibt sich ein Rückgang in der Anzahl der Erwerbspersonen um etwa 2,8 Mio. auf etwa 39,4 Mio. in 2040. Inwieweit dieses Szenario realisiert wird, hängt von dem Ausmaß künftiger Arbeitsmarktreformen und den daraus resultierenden Veränderungen im Erwerbsverhalten der Bevölkerung und der Arbeitsnachfrage ab. Ohne weitere Arbeitsmarktreformen würde die Anzahl der Erwerbspersonen bis 2040 um über 9 Mio. auf 32,6 Mio. zurückgehen.

Zugleich altert die Erwerbsbevölkerung. Das Durchschnittsalter wird bis zum Jahr 2040 von 40 auf knapp 42 Jahre ansteigen. Der Anteil der Erwerbspersonen im Alter 55+ wird von etwa $11 \%$ auf $20 \%$ in 2040 und $21 \%$ in 2050 steigen.

In jedem Fall, auch dem günstigsten, wird sowohl die Erwerbspersonen- als auch die Erwerbstätigenanzahl künftig schrumpfen. Die Wirtschaftspolitik sollte dies im Auge behalten.

\author{
Adresse: \\ Christina Benita Wilke \\ Mannheim Research Institute for the Economics of Aging \\ (MEA) \\ Universität Mannheim \\ L13,17 \\ D-68131 Mannheim \\ E-mail: wilke@mea.uni-mannheim.de
}

Danksagungen: Wir danken Anette Reil-Held und Matthias Weiss für wertvolle Kommentare und Diskussionen, sowie Reinhold Zahn vom Statistischen Bundesamt für detaillierte Auskünfte und zusätzliches Datenmaterial zur 11. Koordinierten Bevölkerungsvorausberechnung. Unser Dank gilt ferner den Förderern des MEA für die Unterstützung bei der Erstellung dieses Beitrags, vor allem dem Land Baden-Württemberg und dem Gesamtverband der Deutschen Versicherungswirtschaft (GDV). 


\section{Szenarien zur mittel- und langfristigen Entwicklung der Anzahl der Erwerbspersonen und der Erwerbstätigen in Deutschland}

Von Axel Börsch-Supan und Christina B. Wilke

\section{Einleitung}

Der demographische Wandel führt dazu, dass es immer mehr ältere und immer weniger jüngere Menschen gibt. Dies hat nicht nur sozialpolitische Konsequenzen, wie sie im Hinblick auf die Nachhaltigkeit der sozialen Sicherungssysteme in den vergangenen Jahren in der Öffentlichkeit verstärkt diskutiert wurden, sondern beeinflusst unser gesamtes Wirtschaftsgeschehen und wird in den kommenden Dekaden einen tiefgreifenden makroökonomischen Strukturwandel hervorrufen. Maßgeblich für das Ausmaß dieser sich abzeichnenden Entwicklung ist jedoch nicht das schlichte Verhältnis der Älteren zu den Jüngeren in einer Gesellschaft. Vielmehr interessiert deren Stellung im Erwerbsleben, d.h. relevant ist die künftige Arbeitsmarktentwicklung.

Dieses Papier legt Projektionen zur mittel- und langfristigen Entwicklung der Anzahl der Erwerbspersonen sowie der Erwerbstätigen in Deutschland vor. Welche Erwerbspersonen- bzw. Erwerbstätigenentwicklung ist dabei realistisch? Wir wählen in diesem Beitrag drei Szenarien, die die Spannbreite möglicher künftiger Entwicklungen aufzeigen. Die Ober- und Untergrenzen sind leicht gesetzt. Nach oben hin begrenzt die durch die Demographie vorgegebene Bevölkerung im erwerbsfähigen Alter die Entwicklungsmöglichkeiten. In unserem optimistischen Szenario gehen wir davon aus, dass dieses Erwerbspersonenpotential bestmöglich ausgeschöpft wird. Die Untergrenze wird durch den Status Quo markiert, d.h. wenn sich die heutigen Erwerbsquoten nicht ändern. Ein Rückwärtstrend der Erwerbsquoten (und somit eine noch niedrigere Untergrenze) erscheint uns hingegen nicht plausibel.

Was ist nun ein realistisches Szenario innerhalb dieser Ober- und Untergrenzen? Um eine Idee davon zu bekommen, wie weit das Erwerbspersonenpotential in realistischem Maße ausgeschöpft werden kann, ist ein Blick auf die europäischen Nachbarländer hilfreich in denen ähnliche Arbeitsmarktreformen wie die deutschen stattfinden. Insbesondere Dänemark eignet sich als ein vorsichtig optimistisches Szenario. Dänemark, aber auch die übrigen skandinavischen Länder und die Schweiz zeichnen 
sich durch eine relativ hohe Erwerbsbeteiligung sowohl der Frauen als auch der Älteren aus. Für unser vorsichtig optimistisches Szenario nehmen wir daher an, dass sich die deutschen Erwerbsquoten im Laufe der nächsten dreißig Jahre nach und nach dem heutigen dänischen Niveau anpassen. Unterstellt man dieses Szenario, so zeigt sich, dass ein Großteil des demographischen Drucks durch den Arbeitsmarkt abgefedert werden kann. Dies ist eine wichtige wirtschaftspolitische Botschaft.

Natürlich sind weitere Szenarien denkbar, vor allem ein Nachlassen der Reformtätigkeit, die jedoch nicht im vollständigen Stillstand unseres Status-Quo-Szenarios endet. Wir modellieren dies mithilfe eines Szenarios, das wir „Agenda 1005“ nennen. Dieses Szenario geht davon aus, dass die in der Agenda 2010 enthaltenen Reformen nur zur Hälfte umgesetzt werden. Weitere Szenarien kann der Leser approximativ durch Interpolation unserer Ergebnisse bilden.

Methodisch gehen unsere Projektionen zurück auf eine Erwerbstätigkeitsprognose für Deutschland von Börsch-Supan (2003). Neuere angebotsorientierte Arbeitsmarktprojektionen wurden für Deutschland 2005 vom Institut für Arbeitsmarkt- und Berufsforschung (IAB) (Fuchs und Dörfler (2005a und 2005b, sowie Fuchs und Söhnlein (2007)), sowie von der Europäischen Kommission herausgegeben (Carone (2005)). Eine eher nachfrageorientierte Arbeitsmarktprojektion erstellte die Kommission für die Nachhaltigkeit in der Finanzierung der Sozialen Sicherungssysteme (2003) („Rürup-Kommission“). Allerdings basieren diese Studien auf älteren Bevölkerungsprognosen. Ziel dieses Beitrags ist es, auf den aktuellsten Bevölkerungsvorausberechnungen des Statistischen Bundesamtes basierend die Spannweite plausibler Erwerbsprojektionen aufzuzeigen und zwei realistische Szenarien vorzustellen.

Der Beitrag gliedert sich in fünf Abschnitte. Abschnitt 2 beschreibt die zugrunde liegenden Bevölkerungsannahmen. Anschließend werden zunächst in Abschnitt 3 die Modellannahmen, Szenarien und die Ergebnisse der Erwerbspersonenprojektionen vorgestellt. Abschnitt 4 geht dann auf die Projektion der Anzahl der Erwerbstätigen ein. Der Beitrag schließt mit einem Fazit und Ausblick in Abschnitt 5.

\section{Annahmen zur künftigen Bevölkerungsentwicklung}

Projektionen der Erwerbspersonen- sowie Erwerbstätigenanzahl hängen maßgeblich von der zugrunde liegenden Bevölkerungsprojektion ab. In diesem Abschnitt werden daher zunächst die Annahmen zur künftigen demographischen Entwicklung vorgestellt. Wir verwenden eigene Bevölkerungsprognosen, die auf der aktuellsten Prognose des statistischen Bundesamtes aufsetzen. Basisjahr unserer Berechnungen ist 
das Jahr 2005. Für die Bevölkerungsprognose gehen wir von einem Prognosezeitraum bis zum Jahr 2050 aus.

11. Koordinierte Bevölkerungsvorausberechnung. Für unsere Berechnungen orientieren wir uns an der 11. Koordinierten Bevölkerungsvorausberechnung des Statistischen Bundesamtes (2006). Als „mittleres Szenario“ werden dort zwei Varianten ausgewiesen: die Variante 1W1 als Unter- und die Variante 1W2 als Obergrenze. Die erste Ziffer beschreibt dabei die zugrunde liegenden Annahmen hinsichtlich der Lebenserwartung, während die zweite Ziffer die Migrationsannahmen ( $\mathrm{W}=$ Wanderungen) beschreibt. Beide Varianten gehen von einer weiterhin stagnierenden Geburtenrate (1,4 Geburten pro Frau) aus. Im Hinblick auf die Lebenserwartung liegt beiden Varianten die sogenannte Basisannahme (1) zugrunde, nach der die Lebenserwartung für Männer auf 83,5 und für Frauen auf 88,0 Jahre im Jahr 2050 ansteigt. ${ }^{1}$ Der Unterschied zwischen den Varianten liegt in den Migrationsannahmen. Während die Variante 1W1 einen Wanderungssaldo von 100.000 (W1) unterstellt und damit den rückgängigen Trend der letzten Jahre aufgreift, basiert die Variante 1W2 auf dem für Deutschland zu beobachtenden langfristigen mittleren Wanderungssaldo von 200.000 (W2) Personen jährlich. Dazwischen gibt es kein weiteres Wanderungsszenario.

MEA-Bevölkerungsprojektionen. Ausgehend von dem Wunsch, ein „mittleres“ Wanderungsszenario zu verwenden und darüber hinaus eine aus unserer Sicht realistischere Entwicklung der Lebenserwartung zu modellieren, erstellen wir zwei eigene Bevölkerungsprojektionen. ${ }^{2}$ Unser Basisszenario MEA 3W1,5 geht von einer deutlich stärkeren Alterung aus. Zweitens verwenden wir ein von der Lebenserwartung der 11. Koordinierten Bevölkerungsvorausberechnung ausgehendes Szenario als Alternative (MEA 1W1,5).

Beide nehmen eine konstante Geburtenrate von 1,4 Geburten pro Frau an. ${ }^{3}$ Der Unterschied zu den Varianten der 11. Koordinierten Bevölkerungsvorausberechnung liegt also lediglich in den Annahmen zum Wanderungssaldo (MEA 1W1,5) und zur Lebenserwartung (MEA 3W1,5). Bei den Wanderungen wählen wir eine mittlere

\footnotetext{
${ }^{1}$ In weiteren Varianten 2W1 bzw. 2W2 stellt das Statistische Bundesamt dieser „Basisannahme“ das Szenario „Hoher Anstieg“ (2) gegenüber, bei dem die Lebenserwartung für Männer auf 85,4 und für Frauen auf 89,8 Jahre im Jahr 2050 ansteigt.

${ }^{2}$ Zu diesem Zweck wurde ein Bevölkerungssimulationsprogramm erstellt und so kalibriert, dass es bei gegebenen Annahmen die Ergebnisse der verschiedenen Varianten der Koordinierten Bevölkerungsvorausberechnung des Statistischen Bundesamtes (2006) reproduziert.

${ }^{3}$ Die Geburtenrate wirkt sich ohnehin erst sehr langfristig auf die Bevölkerungsentwicklung aus und ist daher für unsere Arbeitsmarktprojektionen bis 2050 kaum relevant. Nach 2050 machen sich Änderungen in der Geburtenrate allerdings deutlich bemerkbar. Hinzu kommt, dass eine höhere Geburtenrate letztlich nur im Zusammenhang mit entsprechender Bildung einen positiven Effekt auf den Arbeitsmarkt hat (vgl. hierzu auch Berkel, Börsch-Supan und Ludwig (2002).
} 
Variante zwischen den vom Statistischen Bundesamt ausgegebenen unteren und oberen Varianten und unterstellen einen langfristig mittleren Saldo von 150.000 Personen jährlich (daher die Abkürzung W1,5).

Bei der Lebenserwartung gehen wir für unser Basisszenario (MEA 3W1,5) von einer Trendfortschreibung der vergangenen Jahrzehnte aus. Indem wir den nach dem 2. Weltkrieg beobachtbaren quasi linearen Trend getrennt für Männer und Frauen bis 2050 fortschreiben, ermitteln wir eine Lebenserwartung von 85,7 bzw. 91,7 Jahren für Männer bzw. Frauen im Jahr 2050. Schnabel, Kistowski und Vaupel (2005) berechnen je nach Extrapolationsmethodik eine Lebenserwartung von 92,6 \pm 3,8 Jahre bzw. $94 \pm$ 2,8 Jahre im Jahr 2050. Unsere Annahmen zur Lebenserwartung erscheinen demgegenüber vergleichsweise moderat. Sie liegen aber deutlich über der Basisannahme (1) bzw. leicht über dem Hohen Anstieg (2) des Statistischen Bundesamtes, die wir angesichts der vergangenen stetigen Unterschätzungen für unrealistisch halten. In Anlehnung an die Notation des Statistischen Bundesamtes kennzeichnen wir dieses Lebenserwartungsszenario mit der ersten Ziffer (3).

Ein Überblick über die Annahmen der verschiedenen genannten Bevölkerungsprognosen findet sich in Tabelle 1. Als Vergleich ist dort neben den besprochenen Szenarien des Statistischen Bundesamtes und des MEA auch das Bevölkerungsszenario der Rürup-Kommission angegeben, das nach wie vor vielfach verwendet wird.

TABELLE 1: BEVÖLKERUNGSPROGNOSEN IM ÜBERBLICK

\begin{tabular}{|c|c|c|c|c|}
\hline & $\begin{array}{r}\text { Lebenser } \\
\text { Neugeb }\end{array}$ & $\begin{array}{l}\text { jartung } \\
\text { rener }\end{array}$ & $\begin{array}{l}\text { Wanderungssaldo } \\
\text { (in Tsd.) }\end{array}$ & $\begin{array}{c}\text { Geburtenrate } \\
\text { (Geburten/Frau) }\end{array}$ \\
\hline Rürup-Komm. & M: 81,3 & F: 86,6 & 200 & \multirow{7}{*}{1,4} \\
\hline StatBA $1 \mathrm{~W} 1$ & \multirow{3}{*}{ M: 83,5 } & \multirow{3}{*}{$\mathrm{F}: 88,0$} & 100 & \\
\hline MEA $1 \mathrm{~W} 1,5$ & & & 150 & \\
\hline StatBA $1 \mathrm{~W} 2$ & & & 200 & \\
\hline StatBA $2 \mathrm{~W} 1$ & \multirow{2}{*}{ M: 85,4} & \multirow{2}{*}{$\mathrm{F}: 89,8$} & 100 & \\
\hline StatBA 2W2 & & & 200 & \\
\hline MEA $\quad 3 W 1,5$ & M: 85,7 & $\mathrm{F}: 91,7$ & 150 & \\
\hline
\end{tabular}

Quelle: Eigene Darstellung basierend auf der 11.Koordinierten Bevölkerungsvorausberechnung des Statistischen Bundesamtes (2006) und dem demographischen Szenario der Kommission für die Nachhaltigkeit in der Finanzierung der Sozialen Sicherungssysteme (2003).

Entwicklung der Gesamtbevölkerung. Abbildung 1 zeigt die Entwicklung der Gesamtbevölkerung bis 2050 gemäß den MEA-Bevölkerungsprognosen, den Varianten 1W1 und 2W2 des Statistischen Bundesamtes und dem Szenario der RürupKommission. Interessanterweise entspricht die sich im MEA-Basisszenario (3W1,5) 
ergebende Bevölkerungsentwicklung derjenigen der Variante 1W2 und die des MEA-Alternativszenarios (1W1,5) mit kleinen Abweichungen der Variante 2W1 der 11. Koordinierten Bevölkerungsvorausberechnung. Die Varianten 1W2 und 2W1 werden in Abbildung 1 daher nicht extra dargestellt.

\section{AbBildung 1: BeVÖLKERUngSPRoJeKTiOnen im Vergleich}

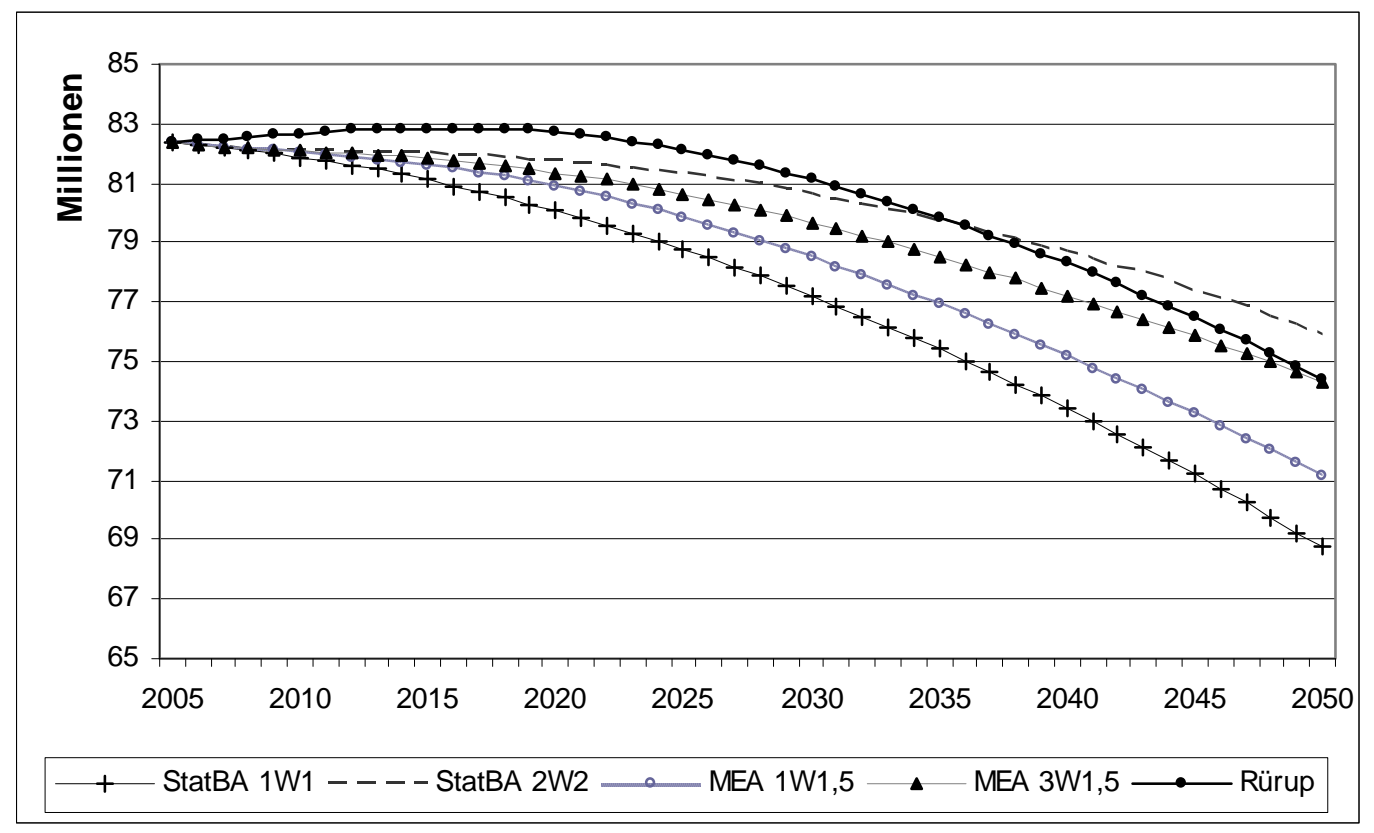

Quelle: Eigene Berechnungen auf Basis von Daten des Statistischen Bundesamtes (www.destatis.de), Daten der 11.Koordinierten Bevölkerungsvorausberechnung des Statistischen Bundesamtes (2006), sowie dem demographischen Szenario der Kommission für die Nachhaltigkeit in der Finanzierung der Sozialen Sicherungssysteme (2003).

Ausgehend von einer Bevölkerung von 82,4 Mio. Einwohnern in 2005 führt das MEA-Basisszenario (MEA 3W1,5) zu einem Rückgang um etwa 8 Mio. auf 74,4 Mio. Einwohner in 2050. Dies entspricht den Werten des Rürup-Szenarios zum Endpunkt unserer Projektionen im Jahr 2050. Das Rürup-Szenario unterstellt im Gegensatz zu den neueren Prognosen jedoch eine Zunahme der Bevölkerung bis 2020. Geringer ist der Bevölkerungsrückgang bis 2050 in der Variante 2W2 des Statistischen Bundesamtes, die eine deutlich höhere jährliche Nettomigration zugrunde legt. Im MEA-Alternativszenario (MEA 1W1,5) ergibt sich ein wesentlich stärkerer Bevölkerungsrückgang um knapp 11,3 Mio. auf 71,1 Mio. Einwohner in 2050. Die Variante 1 W1 des Statistischen Bundesamtes liegt mit 68,8 Mio. in 2050 nochmals um gute 2,3 Mio. darunter.

Veränderungen in der Altersstruktur. Neben dem Ausmaß des Bevölkerungsrückgangs ist vor allem die Veränderung der Altersstruktur für die Entwicklung des Erwerbspersonenpotentials und damit für grundlegende Fragestellungen wie bspw. 
der Entwicklung des Pro-Kopf-Einkommens oder der Nachhaltigkeit der Sozialversicherungssysteme relevant. Abbildung 2 zeigt die Entwicklung des Altersquotienten für die oben vorgestellten Bevölkerungsprognosen. Der Altersquotient ist hier definiert als das Verhältnis der Anzahl der 65-Jährigen und Älteren zur Anzahl der 15bis 64-Jährigen.

\section{Abbildung 2: Entwicklung des Altersquotienten}

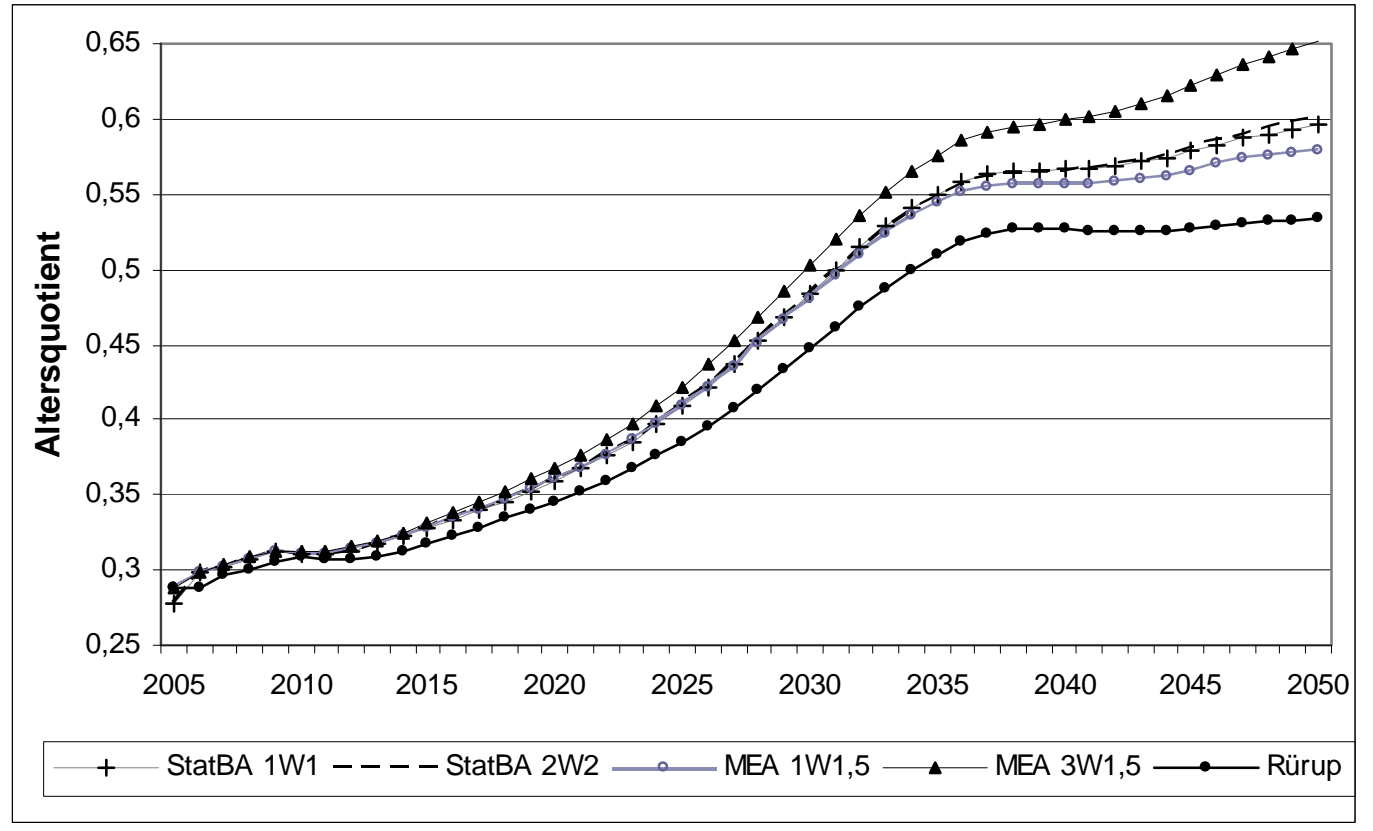

Quelle: Eigene Berechnungen auf Basis von Daten des Statistischen Bundesamtes (www.destatis.de), Daten der 11.Koordinierten Bevölkerungsvorausberechnung des Statistischen Bundesamtes (2006), sowie dem demographischen Szenario der Kommission für die Nachhaltigkeit in der Finanzierung der Sozialen Sicherungssysteme (2003).

Die prozentuale Änderung der Altersstruktur ist wesentlich größer als der prozentuale Bevölkerungsrückgang. Im MEA-Basisszenario (3W1,5) steigt der Altersquotient von 0,29 in 2005 auf 0,65 in 2050, was mehr als einer Verdoppelung entspricht, d.h. einer Person im Alter 65+ werden in diesem von uns als wahrscheinlich eingeschätzten Szenarios im Jahr 2050 noch etwa 1,5 Personen im erwerbsfähigen Alter zwischen 15 und 64 Jahren gegenüber stehen. Der Anstieg des Altersquotienten verläuft im MEA-Alternativszenario (1W1,5) etwas moderater auf 0,58 in 2050. Bei den StatBA-Varianten 1W1 und 2W2 ergibt sich bis 2030 ein nahezu identischer Anstieg des Altersquotienten wie im MEA-Alternativszenario. Der Altersquotient nimmt danach aber nochmals zu, so dass der Altersquotient in 2050 etwas über dem MEAAlternativszenario liegt. Die optimistischste Entwicklung zeigt das Rürup-Szenario, bei dem der Altersquotient bis 2050 „nur“ auf 0,53 ansteigt. 
Im Gegensatz zur Entwicklung der Gesamtbevölkerungszahl, entspricht die Entwicklung des Altersquotienten im MEA-Basisszenario nun der Variante 2W1, während die Altersstruktur im MEA-Alternativszenario relativ mittig bezüglich der beiden Varianten 1W2 und 2W2 der 11. Koordinierten Bevölkerungsvorausberechnung liegt. Dies zeigt, dass sich die MEA-Bevölkerungsprojektionen im Hinblick auf die Altersstruktur deutlich von den Varianten der 11. Koordinierten Bevölkerungsvorausberechnung unterscheiden und macht deutlich, wie sehr die Migrationsannahmen die Altersstruktur insgesamt beeinflussen.

Diese in Abbildung 2 gezeigte Strukturveränderung ist der Kern des Alterungsproblems. Wir betonen, dass er hauptsächlich die Struktur betrifft, viel weniger den Umfang der deutschen Bevölkerung. ${ }^{4}$ Es sei außerdem angemerkt, dass ein moderaterer Bevölkerungsrückgang nicht unbedingt mit einer stärkeren Bevölkerungsalterung und damit einem stärkeren Anstieg des Altersquotienten einhergehen muss, wenn der Anstieg der Lebenserwartung durch eine im Durchschnitt jüngere Altersstruktur beim Wanderungssaldo kompensiert wird. ${ }^{5}$

\section{Projektion der Anzahl der Erwerbspersonen}

Aufbauend auf den Bevölkerungsprojektionen berechnen wir nun die künftige Entwicklung der Anzahl der Erwerbspersonen. Dieser Abschnitt beschreibt den Modellierungsansatz, stellt die ausgewählten Szenarien vor und präsentiert die Ergebnisse der Projektionen. Abschließend betrachten wir die Einzelwirkung der erläuterten Arbeitsangebotsparameter im Rahmen einer Sensitivitätsanalyse.

\subsection{Modellierung}

In einem ersten Schritt legen wir alters- und geschlechtsspezifische Erwerbsquoten ${ }^{6}$ zugrunde, deren Entwicklung im Zeitablauf durch eine Reihe verhaltens- und arbeitsmarktpolitischer Parameter bestimmt wird. In einem zweiten Schritt multiplizie-

4 In Deutschland sind Alterung und Bevölkerungsabnahme gekoppelt. Dies muss nicht so sein, wie das Beispiel der USA zeigt. Hier ist die Geburtenrate deutlich weniger als in Deutschland zurückgegangen, während die Lebenserwartung ähnlich angestiegen ist wie in Deutschland.

${ }^{5}$ Ein Beispiel ist das Rürup-Szenario. Ein zweites Beispiel sieht man sehr schön an den Varianten 1W1 und 2W2 der 11. Koordinierten Bevölkerungsvorausberechnung, die beide Extremszenarien sind, jedoch nahezu den gleichen Altersquotienten aufweisen.

${ }^{6}$ Anteil der Erwerbspersonen an der Bevölkerung. Unsere Erwerbsquoten basieren auf Daten des Mikrozensus des Statistischen Bundesamtes (www.destatis.de). Aufgrund der Untererfassung marginaler Erwerbstätigkeiten im Mikrozensus wird das Niveau der Gesamterwerbstätigkeit dort allerdings unterschätzt. Wir kalibrieren unsere Erwerbsquoten daher so, dass sie multipliziert mit der Bevölkerung für das Basisjahr die Anzahl der Erwerbspersonen gemäß der aggregierten Erwerbsquote der OECD Labour Statistics (http://www.oecd.org/topicstatsportal/0,3398,en_2825_495670_1_1_1_1_ 1,00.html\#499783) korrekt abbilden. 
ren wir diese Erwerbsquoten mit den alters- und geschlechtsspezifischen Belegungszahlen der Bevölkerungsprognose. ${ }^{7}$ Die Szenarien berücksichtigen Veränderungen in drei wesentlichen das Arbeitsangebot bestimmenden Parametern:

- Berufseintrittsalter,

- Frauenerwerbsquote und

- Renteneintrittsalter.

Herabsetzung des Berufseintrittsalters. Wir gehen davon aus, dass sich das mittlere Berufseintrittsalter nach vorne verschiebt, so dass sich die Erwerbsquoten für die frühen Altersjahre erhöhen. Dazu werden die Erwerbsquoten während der Ausbildungsphase bis zum Alter 27 um die je nach Szenario kleinere oder größere Anzahl an Jahren (siehe Abschnitt 3.2) nach vorne verschoben. Zwischen dem Alter 27 und dem neuen Alter, das nun die Ausbildungsphase beendet werden die Erwerbsquoten denen im Alter 27 angeglichen (verschiebt sich das Berufseintrittsalter um $1 \mathrm{Jahr}$ nach vorne, wäre dies bspw. das Alter 26). Das früheste Berufseintrittsalter, für das im Mikrozensus eine Erwerbstätigkeit registriert wird, liegt bei 15 Jahren. Da ein früherer Eintritt in das Berufsleben unrealistisch ist, wird dieses Eintrittsalter beibehalten und es werden ihm lediglich die höheren Erwerbsquoten zugewiesen. Die zeitliche Anpassung der alters- und geschlechtsspezifischen Erwerbsquoten vom Basisjahr bis zum Zieljahr der Prognose erfolgt linear, indem die Differenz zwischen dem Basisjahr und dem Zieljahr gleichmäßig auf alle Prognosejahre aufgeteilt wird.

Erhöhung der Frauenerwerbsquote. Wir nehmen ferner an, dass sich die altersspezifischen Frauenerwerbsquoten im Zeitablauf an die der Männer angleichen. Wir modellieren diese Anpassung, indem wir die Differenz zwischen den Erwerbsquoten der Männer und Frauen um einen bestimmten Faktor verringern. Wieder erfolgt die zeitliche Anpassung zwischen den Erwerbsquoten im Basis- und im Zieljahr linear.

Erhöhung des Renteneintrittsalters. Wir gehen schließlich davon aus, dass sich das mittlere Renteneintrittsalter nach hinten verschiebt, so dass sich die Erwerbsquoten für die späteren Altersjahre erhöhen. Dazu werden die Erwerbsquoten ab dem Alter 47, zu dem die altersspezifischen Erwerbsquoten ihren Höhepunkt erreicht haben, um die dem jeweiligen Szenario entsprechende Anzahl an Jahren nach hinten verschoben. Zwischen dem Alter 47 und dem neuen Alter, ab dem nun nach und nach der Übergang in die Rente statt werden die Erwerbsquoten denen im Alter 47 angeglichen findet (verschiebt sich das mittlere Renteneintrittsalter um 1 Jahr nach hinten, wäre dies bspw. das Alter 48). Das mittlere Renteneintrittsalter liegt derzeit

\footnotetext{
${ }^{7}$ Wir verzichten hier bewusst auf eine Differenzierung nach Ost und West, nicht zuletzt, weil eine substanzielle Vorausschätzung der entsprechenden Parameter schwierig erscheint. Zu Unterschieden im Erwerbsverhalten zwischen Ost und West, siehe bspw. Fuchs und Söhnlein (2007).
} 
bei etwa 62 Jahren, so dass eine Verschiebung um 2 Jahre ein mittleres Renteneintrittsalter von etwa 64 Jahren bedeutet. Die zeitliche Anpassung der alters- und geschlechtsspezifischen Erwerbsquoten vom Basisjahr bis zum Zieljahr der Prognose erfolgt wiederum linear.

Eine Änderung einer oder mehrerer dieser Parameter hat einen direkten Einfluss auf die Höhe der alters- und geschlechtsspezifischen Erwerbsquoten und wirkt somit unmittelbar auf das Arbeitsangebot.

\subsection{Szenarien}

Mit Hilfe der im vorigen Abschnitt erläuterten Parameter lässt sich nahezu jede künftige Arbeitsangebotsentwicklung modellieren. Welche künftige Arbeitsangebotsentwicklung ist nun als realistisch zu betrachten? Welche Spannbreite kann sich aufgrund politischen Handelns bzw. politischer Untätigkeit ergeben? Im Folgenden stellen wir vier Szenarien vor („Status Quo“, „Best Case“, „Dänemark“, „Agenda 1005“), die verdeutlichen, wie unterschiedlich sich das Arbeitsangebot entwickeln kann, je nachdem welche Veränderungen für das Erwerbsverhalten der Bevölkerung angenommen werden.

Szenario „Status Quo“. Im Szenario „Status Quo“ wird davon ausgegangen, dass die heutigen Erwerbsquoten auch in Zukunft bestehen bleiben. Dieses Szenario unterstellt folglich, dass sich das mittlere Berufseintrittsalter nicht nach vorne verschiebt und sich die Ausbildungsdauer nicht verkürzt, dass sich die Frauenerwerbsquote nicht an die der Männer angleicht und dass die aktuellen Reformen im Bereich der Rentenversicherung keine weitere Erhöhung des mittleren Rentenzugangsalters bewirken. Wir halten diese Entwicklung für sehr unwahrscheinlich. Sie ist übermäßig pessimistisch und soll lediglich aufzeigen, wie hoch der demographische Druck auf das Arbeitsangebot werden kann.

Szenario „Best Case“. Zeigt das Status-Quo-Szenario die Untergrenze potentieller künftiger Arbeitsangebotsentwicklungen auf, so beschreibt das Best-Case-Szenario die Obergrenze. Es wird davon ausgegangen, dass sich bis 2040 das mittlere Berufseintrittsalter um 2 Jahre nach vorne verschiebt, dass sich die Frauenerwerbsquote nahezu vollständig (um 90\%) an die der Männer angleicht, die Erwerbsquote der Männer sich im Gegenzug auf 95\% reduziert und dass sich das mittlere Renteneintrittsalter um 3 Jahre nach hinten verschiebt. Dieses Szenario halten wir für äußerst optimistisch. Es soll verdeutlichen, welche Entwicklung unserer Meinung nach auf dem Arbeitsmarkt zu erzielen wäre, wenn man in Deutschland z.B. USamerikanische Beschäftigungsquoten erreichen würde. 
Szenario „Dänemark“. Dieses Szenario orientiert sich an den heutigen alters- und geschlechtsspezifischen Erwerbsquoten in Dänemark. Die Unterschiede zwischen den heutigen deutschen (D) und dänischen (DK) Erwerbsquoten zeigt Abbildung 3. Sie zeigt, dass die dänischen Erwerbsquoten der Frauen in allen Altersjahren deutlich oberhalb denen der deutschen Frauen liegen. Bei den Männern sind die Erwerbsquoten beider Länder in den jungen Jahren etwa gleich. In den späteren Altersjahren bis zum Renteneintritt liegen die Erwerbsquoten der dänischen Männer hingegen deutlich oberhalb der Erwerbsquoten der deutschen Männer.

AbBILDUNG 3: DEUTSCHE UND DÄNISCHE ERWERBSQUOTEN IM VERGLEICH

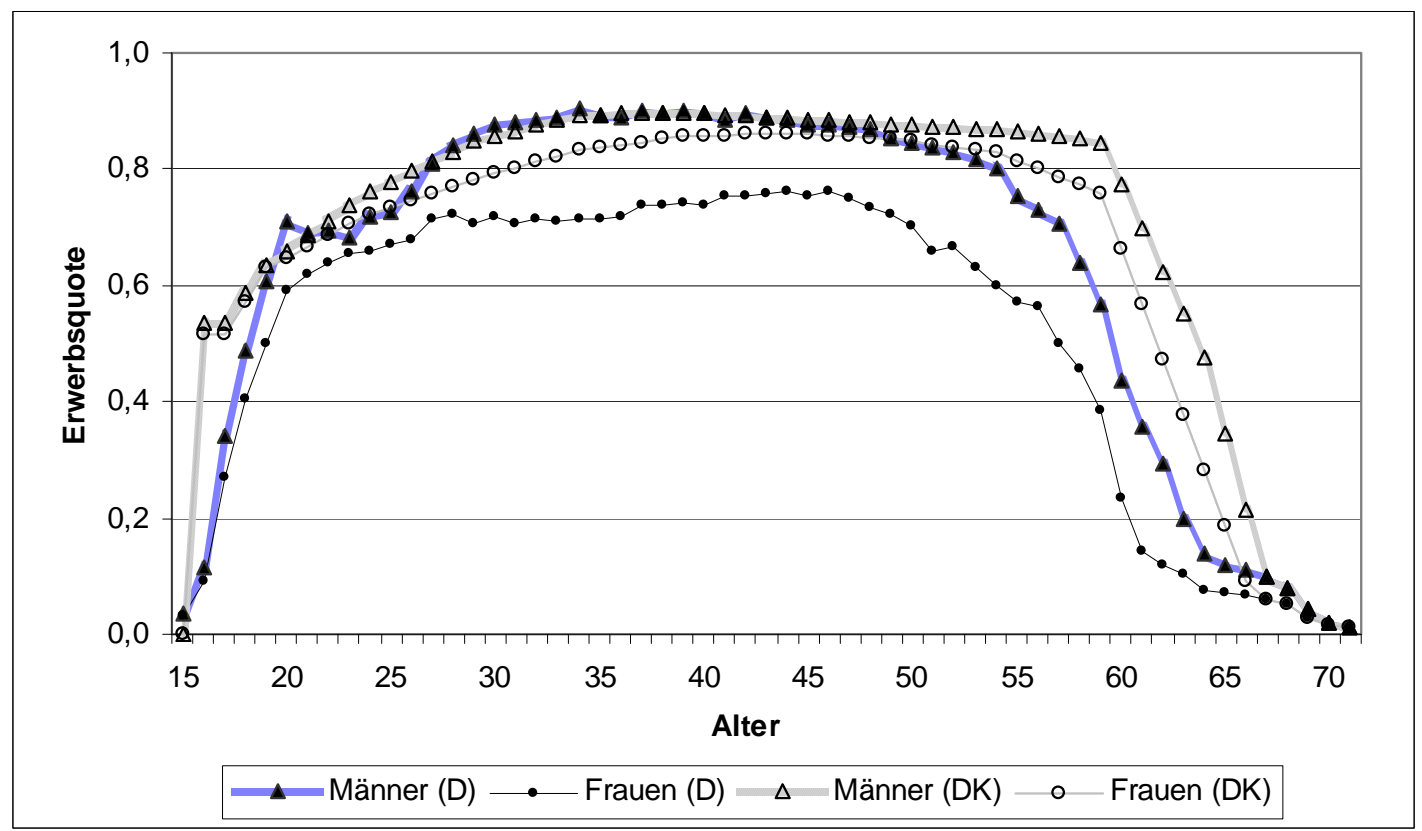

Quelle: Eigene Berechnungen auf Basis von Daten aus dem Mikrozensus des Statistischen Bundesamtes (www.destatis.de) und Statistics Denmark (www.statbank.dk).

Im Dänemark-Szenario wird von einer Angleichung der deutschen an die dänischen Erwerbsquoten ausgegangen. Geht man von einer Angleichung bis zum Jahr 2040 aus, so entspricht die daraus resultierende künftige Arbeitsangebotsentwicklung einer Entwicklung, wie sie bei einer Herabsetzung des mittleren Berufseintrittalters um ein Jahr, einer Angleichung der Frauenerwerbstätigkeit an die der Männer um 90\% bei entsprechender Reduktion der Männererwerbstätigkeit auf 95\% und einer Heraufsetzung des mittleren Renteneintrittsalters um 2 Jahre bis 2040 zu erwarten wäre. Dieses Szenario bildet unserer Meinung nach eine realistische Entwicklung ab, sofern die Reformen der Agenda 2010 konsequent fortgeführt werden. Es ist zu betonen, dass diesem Szenario die eher moderate Annahme zugrunde liegt, dass Deutschland in den kommenden 30 Jahren diejenigen Arbeitsmarktreformen durchführen kann, 
die Dänemark in den letzten 10 Jahren durchgeführt hat, also gerade einmal ein Drittel des dänischen Reformtempos durchhält.

Szenario „Agenda 1005“. Ob die mit der Agenda 2010 avisierten Reformen im politischen System Deutschlands tatsächlich durchgehalten werden, ist nicht sicher. Wir modellieren daher noch ein viertes Szenario, in dem wir davon ausgehen, dass die Agenda 2010 nur zur Hälfte realisiert wird. Wir nennen dieses Szenario daher „Agenda1005“. Wir unterstellen in diesem Fall bis zum Jahr 2040 eine Erhöhung des mittleren Renteneintrittsalters um nur noch 1 Jahr. Dies modellierte eine Unterhöhlung der Heraufsetzung des normalen Rentenalters von 65 auf 67 Jahre durch eine erleichterte Erwerbsminderungsrente und ähnliche Frühverrentungsmechanismen. Wir gehen in diesem Szenario ferner von einer Angleichung der Frauenerwerbsquote um lediglich 75\% an die der Männer aus, während die Erwerbsquoten der Männer unverändert bleiben. Schließlich unterstellt das Szenario eine Verschiebung des mittleren Berufseintrittsalter um 1 Jahr nach vorne, die eine flächendeckende Einführung des achtjährigen Gymnasiums und der BA/MA-Studiengänge reflektieren.

Die vier ausgewählten Szenarien zeigen somit zum einen die mögliche Spannbreite künftiger potentieller Arbeitsangebotsentwicklungen auf, zum anderen enthalten sie zwei realistische Szenarien, die sich an der heutigen Arbeitsmarktsituation in Dänemark bzw. den aktuellen Reformbemühungen in Deutschland orientieren.

\subsection{Ergebnisse}

Unsere Simulationen gehen vom Basisjahr 2005 aus, für das die letzten historischen Bevölkerungsdaten vorliegen. Sie beinhalten bis zum Jahr 2008 jedoch noch keinerlei Veränderungen in den Erwerbsquoten, da die für die Szenarien „Dänemark“ und „Best Case“ unterstellten und über die Agenda 2010 hinausgehenden Arbeitsmarktreformen prinzipiell erst ab 2008 Wirkung zeigen können. Die Projektionen gehen bis zum Jahr 2040. Danach halten wir die Erwerbsquoten konstant. Veränderungen im Arbeitsangebot begründen sich dann nur noch durch die zugrunde liegende Demographie. Um auch diese Entwicklung aufzuzeigen, stellen wir unsere Ergebnisse der Projektionen in den Abbildungen bis zum Jahr 2050 dar. Die zugrunde liegende Bevölkerungsprojektion ist unser Basisszenario MEA 3W1,5.

Entwicklung der Anzahl der Erwerbspersonen. Die Anzahl der Erwerbspersonen geht in allen vier Szenarien zurück (siehe Abbildung 4), wenn auch das Ausmaß des Rückgangs sehr unterschiedlich ausfällt. Während im Status Quo ein Rückgang von 9,6 Mio. (etwa 22,7\%) auf etwa 32,6 Mio. Erwerbspersonen in 2040 zu verzeichnen ist, sinkt die Anzahl der Erwerbspersonen bis 2040 im Szenario Agenda 1005 um 5,8 Mio. auf 36,4 Mio. (etwa 13,7\%), im Dänemark-Szenario um 3,9 Mio. (etwa 9,2\%) 
auf knapp 38,3 Mio. und im Best-Case-Szenario um 2,8 Mio. auf nur 39,4 Mio.(etwa 6,6 \%). Bis 2040 wird es im Dänemark-Szenario somit fast 4 Millionen weniger Erwerbspersonen als im Jahr 2005 geben. Das Arbeitsangebot wird also, absolut gesehen, um etwa die heutige Zahl der Arbeitslosen schrumpfen.

\section{AbBildung 4: ENTWiCKLung Der ANZAHL DER ERWERBSPERSONEN}

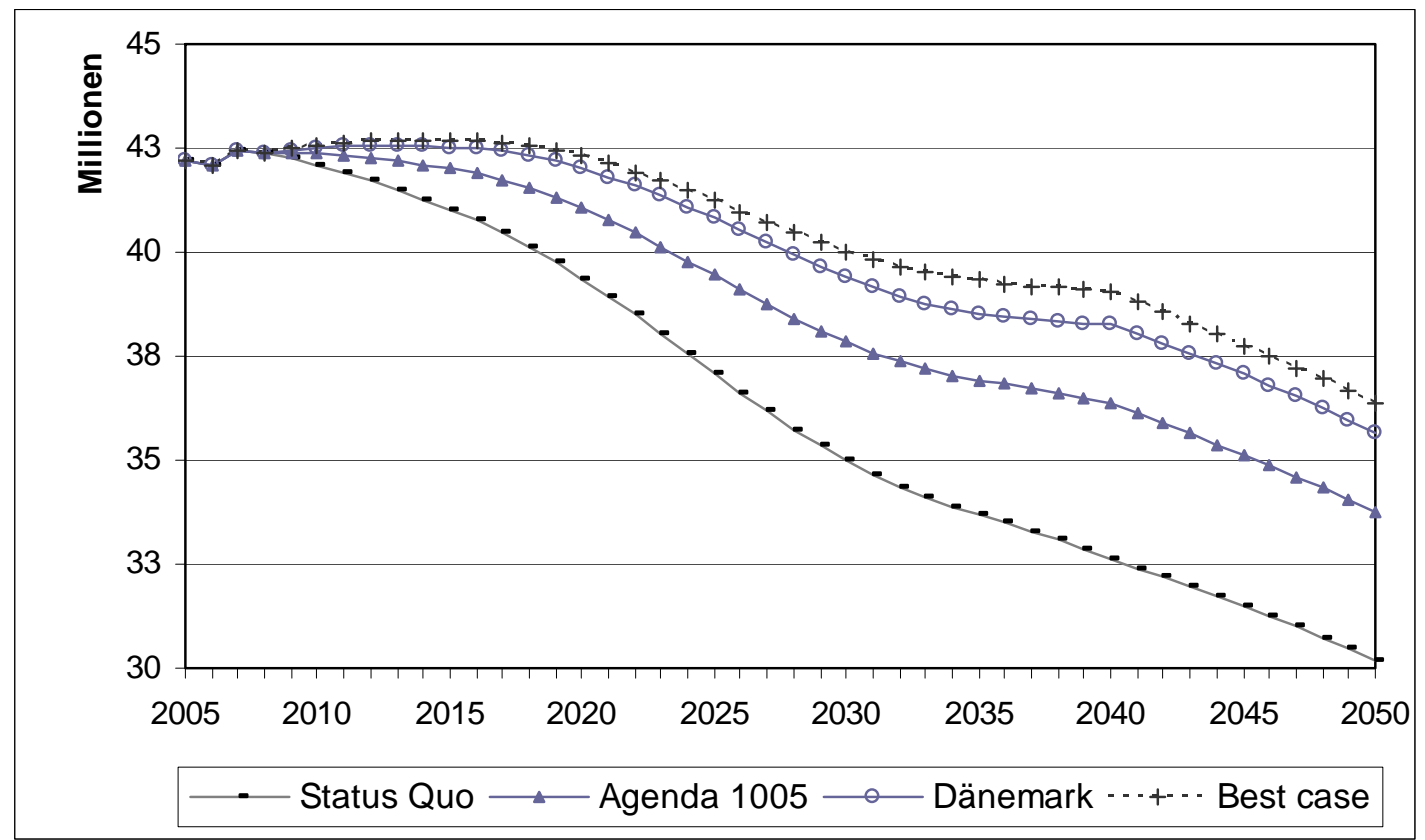

Quelle: MEA: Eigene Berechnungen

Ab 2040 nimmt der Rückgang der Erwerbspersonenanzahl zu und entspricht dem Tempo im Status Quo. In den Szenarien „Best Case“ und „Dänemark“ entsteht ein Knick, da die Erwerbsquoten nach 2040 annahmegemäß ihren langfristigen Wert erreicht haben, also nach 2040 nicht weiter steigen, die Bevölkerung aber weiterhin sinkt.

Selbst wenn das Erwerbspersonenpotenzial also wie im Best-Case-Szenario so gut wie möglich ausgeschöpft wird, wird sich der Bevölkerungsrückgang langfristig auch in einem deutlich weiteren Rückgang der Erwerbspersonen auf dem Arbeitsmarkt zeigen - allerdings auf einem wesentlich höheren Niveau mit gut 5,4 Mio. Erwerbspersonen mehr als im Status Quo.

Altersstruktur. Nicht nur die Anzahl, sondern auch die Altersstruktur der Erwerbpersonen wird sich in den nächsten Dekaden deutlich ändern. Abbildung 5 zeigt die Altersverteilung der Erwerbspersonen im Dänemark-Szenario für die Jahre 2010, 2020, 2030, 2040 und 2050. 
Abbildung 5: Altersstruktur der Erwerbspersonen

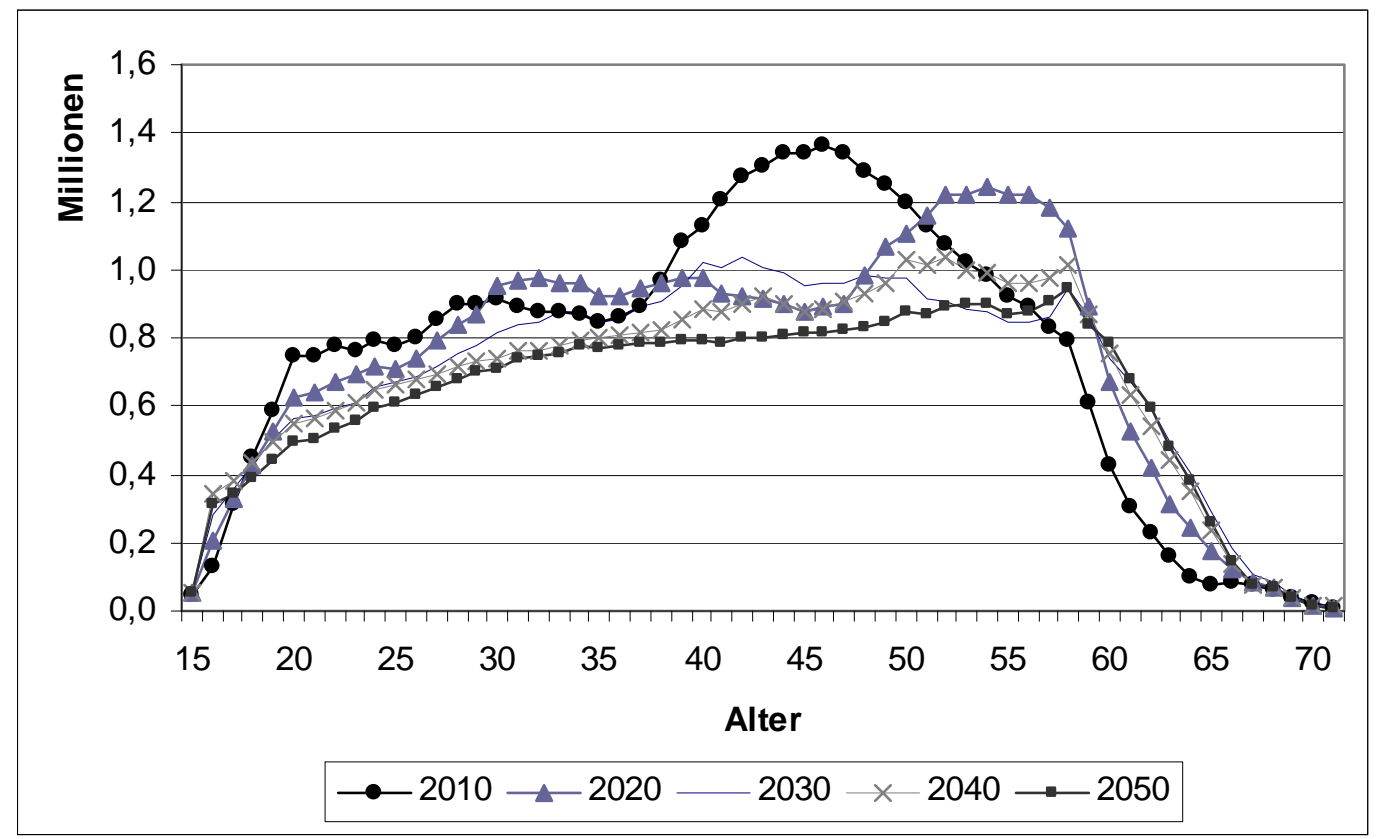

Quelle: MEA: Eigene Berechnungen

Man sieht deutlich, wie der Gipfel der Verteilung sich zunächst nach rechts bewegt und dann zwischen 2020 und 2030, wenn die Baby-Boomer in Rente gehen, verschwindet. Danach wird die Altersverteilung flacher und verändert sich in den Folgejahren nur noch wenig.

Durchschnittsalter. Das durchschnittliche Alter der Erwerbspersonen wird im Dänemark-Szenario in den nächsten 20 Jahren von 39,8 auf etwa 41,7 in 2025 ansteigen, dann für 10 Jahre lang wieder leicht rückläufig sein und schließlich bis 2050 um ein weiteres Vierteljahr auf etwa 42 Jahre ansteigen (Abbildung 6). Im Best-CaseSzenario ist die Entwicklung ähnlich. In den Szenarien Agenda 1005 und Status Quo hingegen fällt der Rückgang des Durchschnittsalters nach 2025 wesentlich stärker aus, weil dann der Großteil der Baby-Boom-Generation bereits in Rente gegangen sein wird. 


\section{AbBildung 6: DurchschnitTsalter Der ERWERBSPERSONEN}

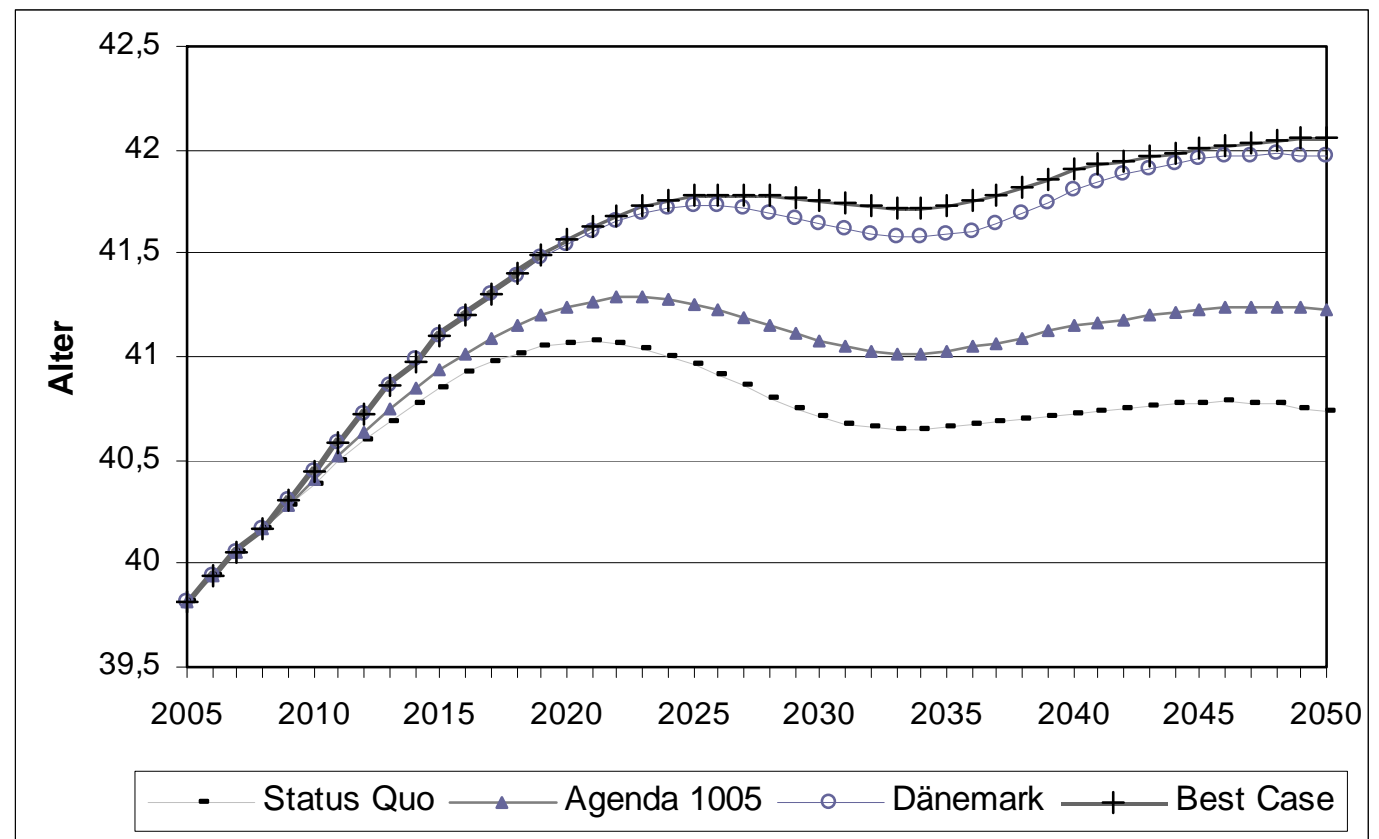

Quelle: MEA: Eigene Berechnungen

Erwerbsbeteiligung Älterer. Dieser Altersstrukturwandel wird noch deutlicher, wenn man den Anteil der Erwerbspersonen im Alter 55+ betrachtet (Abbildung 7). Hier sieht man die temporäre "Verjüngung" der Erwerbspersonen, wenn die Babyboomgeneration in Rente gegangen sein wird, noch deutlicher als oben im Status Quo Szenario.

\section{Abbildung 7: Anteil der ErWerbspersonen im Alter 55+}

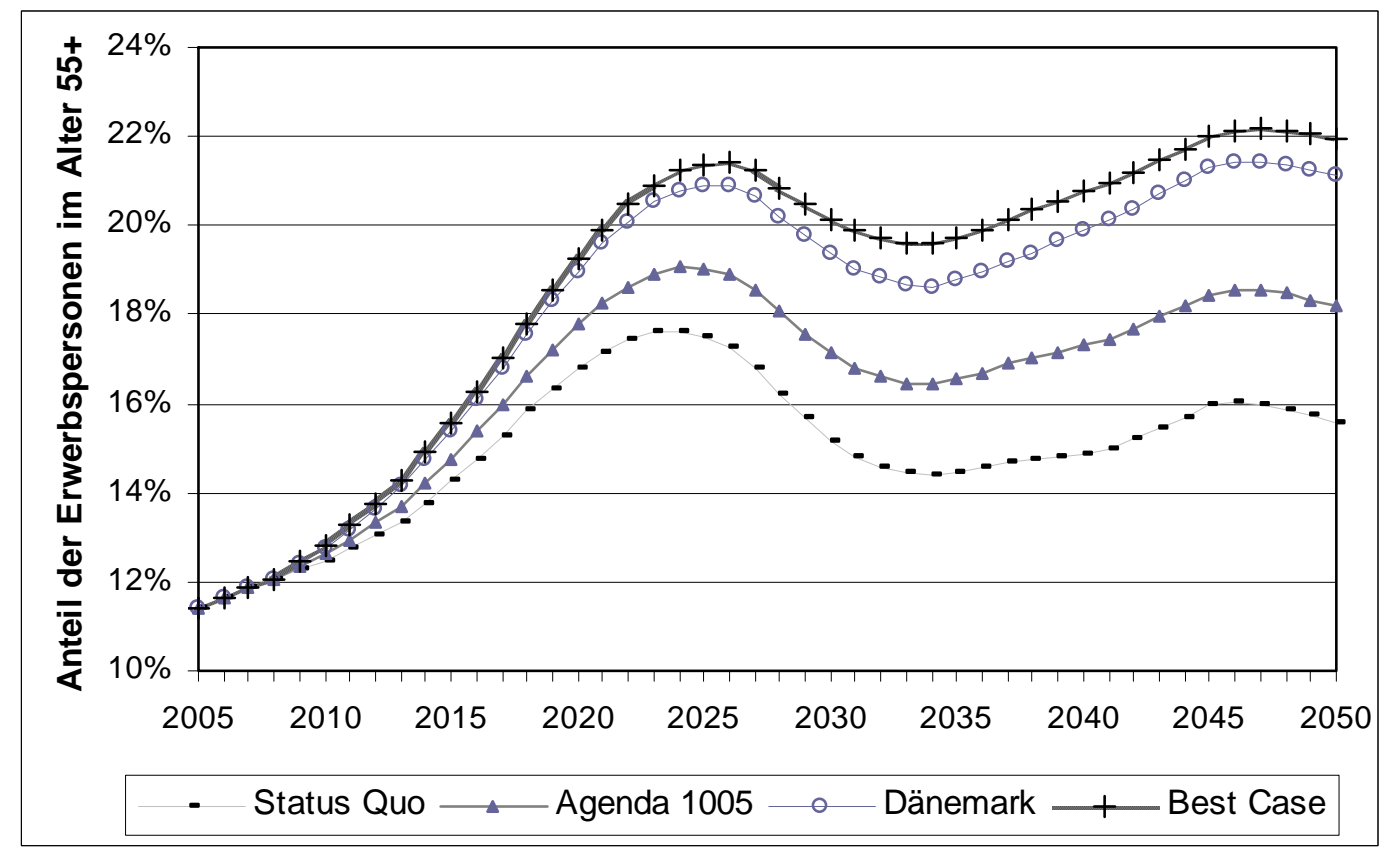

Quelle: MEA: Eigene Berechnungen 
Es wird zudem deutlich, dass die Altersstrukturverschiebung kein Übergangsphänomen, sondern eine permanente Veränderung ist. Im Dänemark-Szenario und im BestCase-Szenario verdoppelt sich der Anteil der Erwerbspersonen im Alter 55+ bis 2050 nahezu. Im Szenario Agenda 1005 nimmt der Anteil dieser Erwerbspersonen immerhin immer noch um fast zwei Drittel zu. Diese Entwicklung zeigt, dass wir uns auf eine solche Alterstrukturverschiebung in jedem Fall einstellen müssen, wenn ein stärkerer Rückgang in der Anzahl der Erwerbspersonen verhindert werden soll.

\subsection{Sensitivitätsanalyse}

In diesem Abschnitt untersuchen wir die Sensitivität unserer Ergebnisse im Hinblick auf zwei ausgewählte Aspekte. Erstens zeigen wir, inwieweit sich unsere Ergebnisse ändern, wenn den Berechnungen andere Bevölkerungsprojektionen als unser MEABasisszenario (3W1,5) zugrunde gelegt werden. Zweitens haben wir im DänemarkSzenario eine Anpassung bis zum Jahr 2040 unterstellt. Wir zeigen, welche Auswirkungen eine schnellere bzw. langsamere Anpassung auf unsere Ergebnisse hat.

Konsequenzen eines stärkeren Bevölkerungsrückgangs. Inwieweit ändern sich diese Ergebnisse, wenn eine pessimistischere Bevölkerungsprojektion unterstellt wird? Abbildung 8 zeigt die Entwicklung der Anzahl der Erwerbspersonen im Dänemark-Szenario für beide MEA Bevölkerungsprojektionen sowie der Variante 1W1 der 11. Koordinierten Bevölkerungsvorausberechnung im Vergleich.

\section{Abbildung 8: Entwicklung der Anzahl der ERWERbSPERSonen bei ALTERNATIVEN BEVÖLKERUNGSPROGNOSEN}

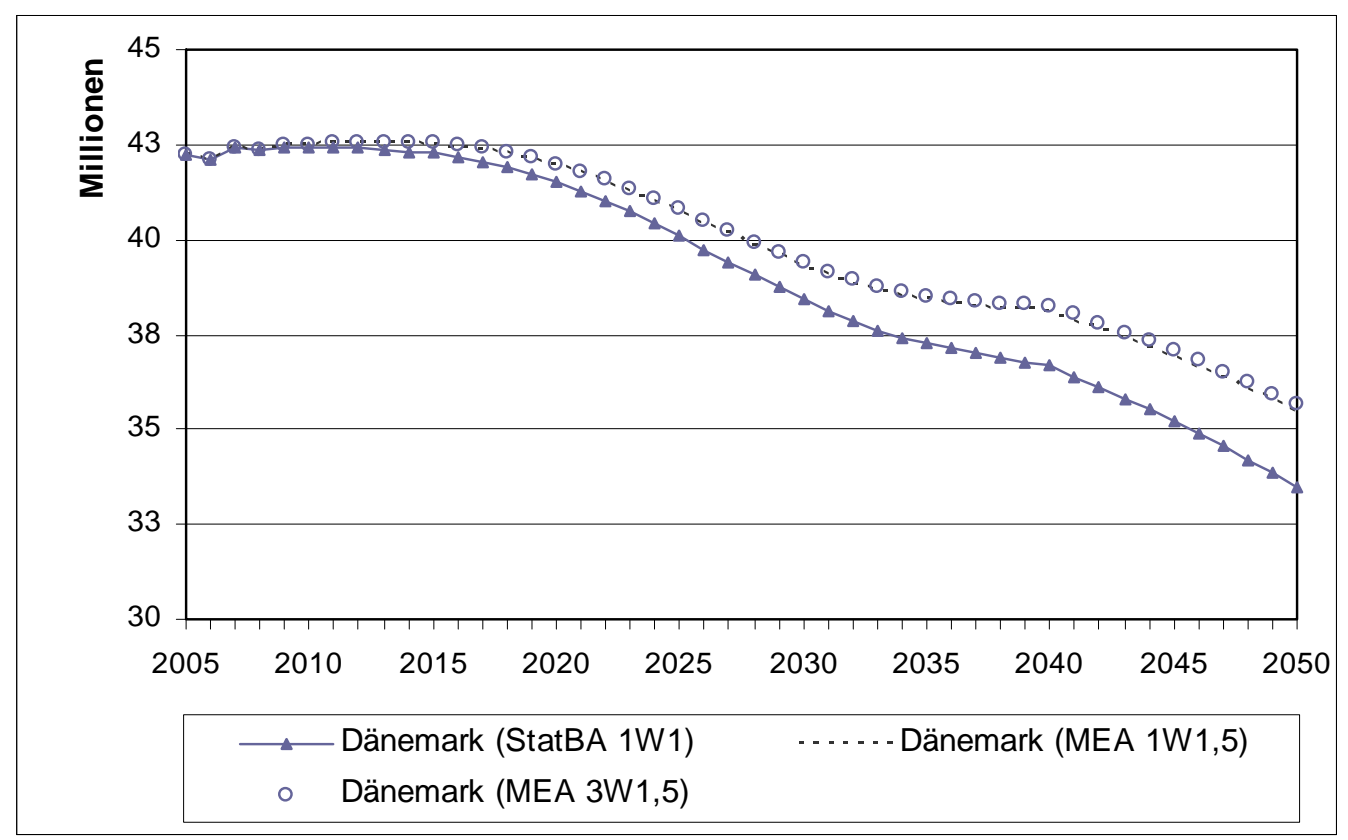

Quelle: MEA: Eigene Berechnungen 
Da sich die Bevölkerungsprojektionen MEA 3W1,5 und MEA 1W1,5 nur durch unterschiedliche Annahmen zur Lebenserwartung unterscheiden, sind die Unterschiede hier minimal. Anders sieht dies bei der Variante 1W1 des Statistischen Bundesamtes aus. Hier entwickelt sich die Anzahl der Erwerbspersonen im Dänemark-Szenario deutlich unterhalb der Entwicklung bei zugrunde liegenden MEABevölkerungsprojektionen (etwa 1,6 Mio. in 2040). Der Grund liegt in den moderateren Migrationsannahmen (100 000 anstatt 150 000), die zu einem geringeren Erwerbspersonenpotential bei den Jüngeren führen. Analog dazu führt eine Nettomigration von 200000 anstatt 150000 zu einer um etwa 1,6 Mio. höheren Erwerbsbevölkerung in 2040. Es sei angemerkt, dass selbst ein höherer Wanderungssaldo zwar die Anzahl der Erwerbspersonen generell erhöhen ${ }^{8}$, der allgemeine Trend eines Rückgangs der Erwerbspersonenzahl jedoch auch dadurch letztlich nicht aufgehalten werden kann.

Variation des Dänemark-Szenarios. Im Dänemark-Szenario gehen wir von einer Anpassung der Erwerbsquoten bis 2040 aus. Vollzieht sich diese Anpassung schneller bzw. langsamer, verläuft die resultierende Arbeitsangebotsentwicklung mittelfristig deutlich anders (siehe Abbildung 9).

\section{Abbildung 9: Verschiedene AnpassungszeiträUme im Dänemark- SZENARIO}

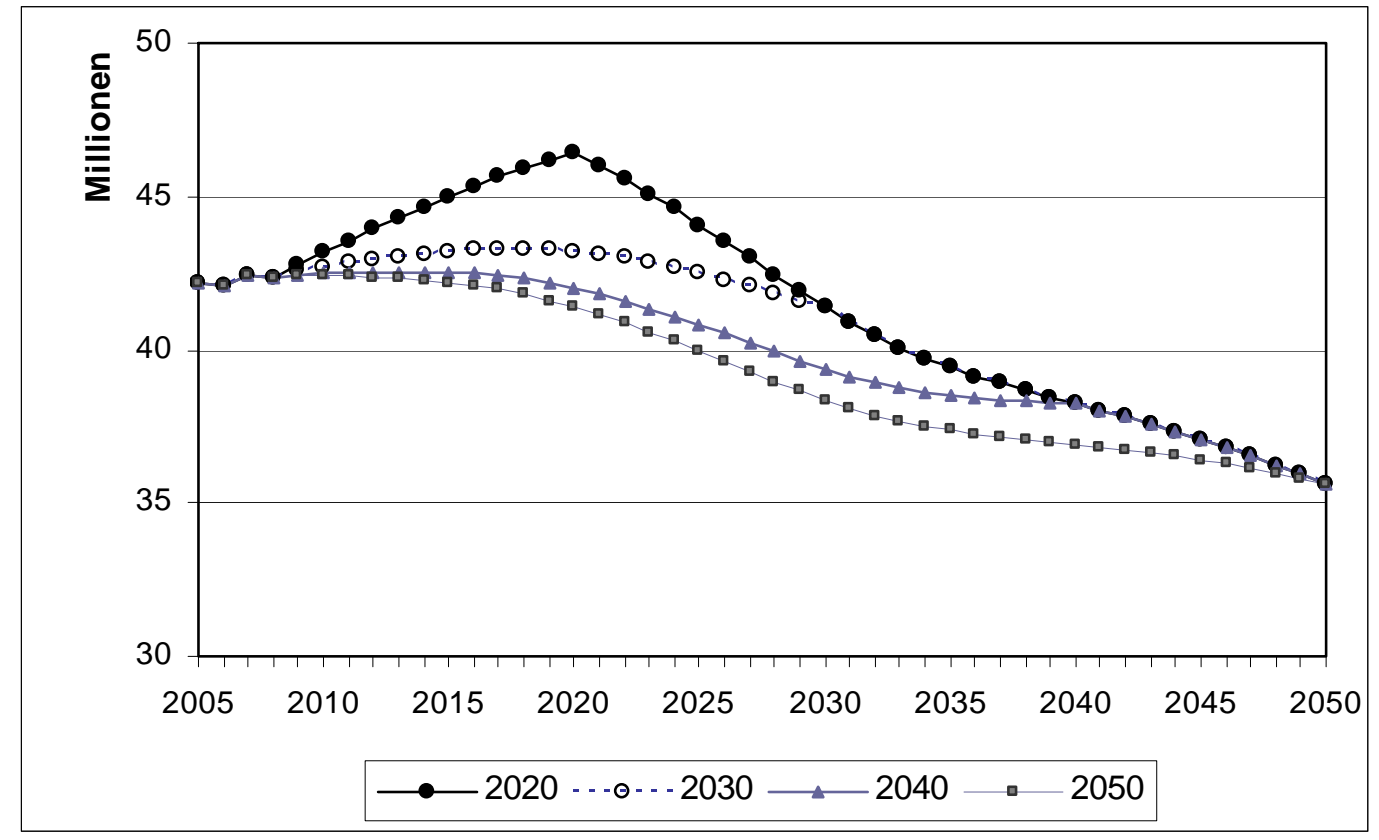

Quelle: MEA: Eigene Berechnungen

\footnotetext{
${ }^{8}$ Zur Rolle der Zuwanderung in der Debatte um die Auswirkungen des demographischen Wandels, siehe bspw. Börsch-Supan (2002a).
} 
Bei einer Anpassung der deutschen Erwerbsquoten an die dänischen bereits bis zum Jahr 2020, würde die Anzahl der Erwerbspersonen auf nahezu 46,5 Mio. steigen, was einem Zuwachs von über 4 Mio. entspricht. Dieser Zuwachs begründet sich durch den starken Anstieg der Erwerbsquoten (die sich über einen kürzeren Zeitraum angleichen) bei einem noch recht verhaltenen Bevölkerungsrückgang. Allerdings ist dieser Zuwachs an Erwerbspersonen nicht nachhaltig, denn nach 2020 geht die Anzahl der Erwerbspersonen wieder zurück und zwar gemäß dem gleichen Trend wie auch in den anderen Szenario-Varianten bei Konstanz der Erwerbsquoten. Hier bestimmt allein die Bevölkerung und die Alterstruktur die Entwicklung in der Anzahl der Erwerbspersonen. Bei einer Anpassung bis 2030 lässt sich dieser extreme „Knick“ nicht mehr beobachten. Stattdessen steigt die Anzahl der Erwerbspersonen nur noch leicht auf gut 43 Mio. in 2020, sinkt bis 2030 nur knapp unter das heutige Niveau und entwickelt sich danach gemäß dem Trend. Eine langsamere Anpassung bis zum Jahr 2050 hingegen führt zwischen 2020 und 2045 zu einer um teils über eine Mio. niedrigeren Erwerbstätigenzahl als bei einer Anpassung bis 2040.

\subsection{Wirkungsanalyse der Arbeitsangebotsparameter}

Unsere Arbeitsangebotsprojektionen basieren auf vier ausgewählten Szenarien, die die Spannbreite möglicher künftiger Entwicklungen sowie zwei realistische Entwicklungen aufzeigen. Ihnen liegen unterschiedliche Annahmen bezüglich der das Arbeitsangebot bestimmenden Parameter (Berufseintrittsalter, Frauenerwerbstätigkeit und Renteneintrittsalter.) zugrunde. Wie groß ist nun der Wirkungsgrad dieser einzelnen Parameter, d.h. welche Parameter haben schon bei kleinen Änderungen einen sichtbaren Einfluss auf das Arbeitsangebot und welche erfordern größere Anpassungen? Nachfolgend betrachten wir zunächst einzeln die Auswirkungen einer Veränderung der jeweiligen Parameter vom Status Quo und diskutieren ob eine solche Veränderung als eher klein bzw. groß einzustufen ist. Danach setzen wir die verschiedenen Parameter in Vergleich.

Abbildung 10 zeigt, welche Auswirkung eine Herabsetzung des Berufseintrittsalters (BEA) um ein Jahr bzw. 2 Jahre bis 2040 auf die Erwerbsquoten und damit die Anzahl der Erwerbspersonen am Arbeitsmarkt hat. Die Anzahl der Erwerbspersonen im Status Quo wird dazu auf Eins normiert. 


\section{ABBILDUNG 10: BERUfSEINTRITTSALTER UND ERWERBSPERSONENANZAHL}

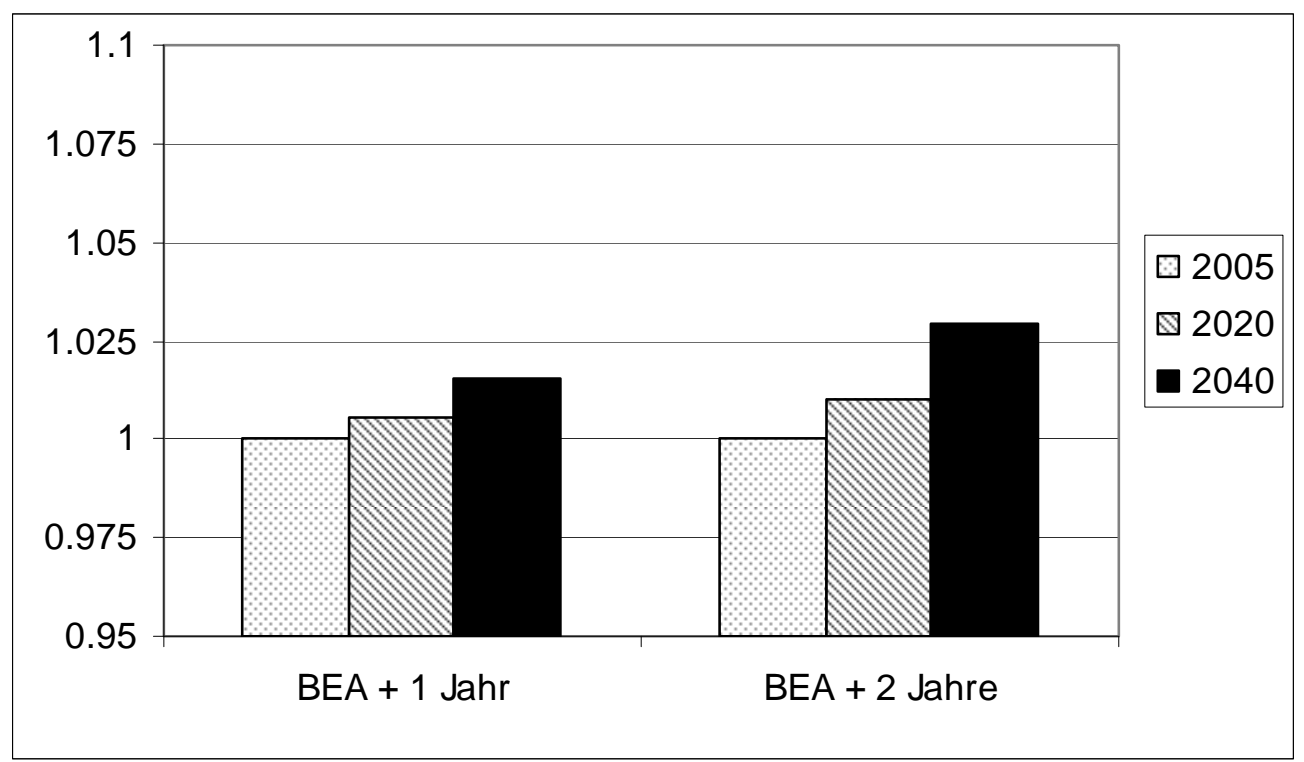

Quelle: MEA: Eigene Berechnungen

Eine Herabsetzung des Berufseintrittsalters um ein Jahr bis 2040 bewirkt demnach eine Steigerung der Anzahl der Erwerbspersonen um 0,7\% bis zum Jahr 2020 und 1,5\% bis 2040 im Vergleich zum Status Quo. Wird das Berufseintrittsalter um 2 Jahre nach vorne verschoben, so verdoppelt sich der Effekt auf 1,4\% bis zum Jahr 2020 und $3,0 \%$ bis 2040 .

Im Hinblick auf die Diskussion um kürzere Ausbildungszeiten ist ein um ein Jahr nach vorne verschobener Berufseintritt künftig sicherlich realistisch. Eine Verschiebung des Berufseintrittsalters um mehr als 2 Jahre nach vorne hingegen erscheint unplausibel, da die Ausbildungszeiten dann unter die kürzesten in den EUMitgliedsländern fallen würden. Die Auswirkungen auf die Erwerbspersonenanzahl sind insgesamt eher gering, da die Jahrgangsstärken niedrig sind. Allerdings ist zu berücksichtigen, dass ein früherer Einstieg der Jüngeren in den Arbeitsmarkt die Altersstruktur der Erwerbspersonen verjüngt.

Erhöhung der Frauenerwerbsquote. Eine Anpassung der Frauenerwerbsquote (FEWT) an die der Männer hingegen kann sehr große Auswirkungen auf die Anzahl der Erwerbspersonen haben, wie Abbildung 11 zeigt. Demnach bewirkt eine Anpassung der Frauenerwerbstätigkeit an die der Männer um 20\% bis 2040 bereits eine Erhöhung der Anzahl der Erwerbspersonen um 1\% bis 2020 und 2\% bis 2040. Eine größere Anpassung um 40\%, 60\% oder 80\% der Differenz zwischen den Erwerbsquoten der Frauen und Männer führt entsprechend zu einer Erhöhung der Erwerbspersonenanzahl um 2\%, 3\% und 4\% bis 2020 und 4\%, 6\%, 8\% bis 2040. Ebenso wie beim Berufseintritt sind die Auswirkungen der einzelnen Änderungen somit im Wesentlichen proportional. 
ABbiLdUng 11: FrAUENERWERBSTÄTIGKEIT UND ERWERBSPERSONENANZAHL

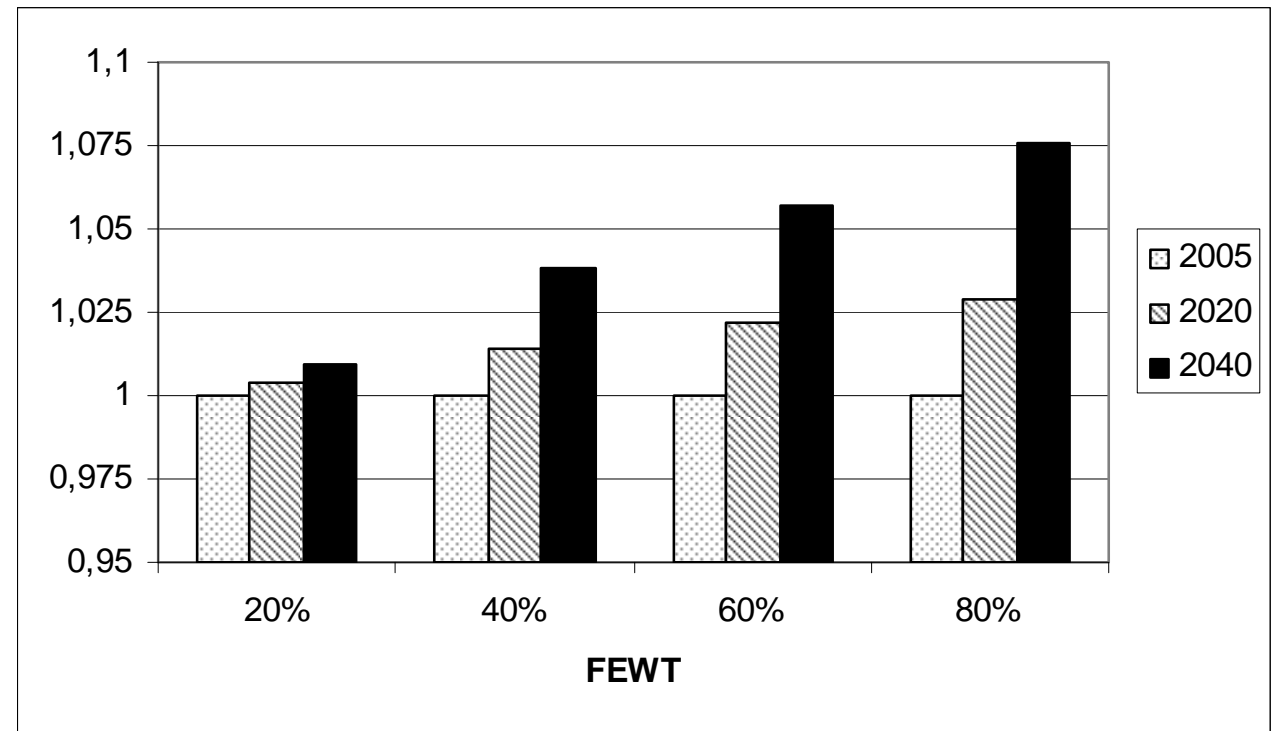

Quelle: MEA: Eigene Berechnungen

Eine Angleichung der Frauenerwerbsquoten an die der Männer hat somit eine relative große Wirkung. Da Deutschland im Vergleich mit anderen Ländern wie bspw. Dänemark derzeit noch eine eher geringe Erwerbsbeteiligung von Frauen verzeichnet (vergleiche Abbildung 3 oben), besteht hier noch Spielraum für künftige Anpassungen. Voraussetzung ist allerdings eine entsprechende Politik, die die Vereinbarkeit von Familie und Beruf weiter fördert und Abschied von den klassischen Geschlechterrollen nimmt.

Erhöhung des Renteneintrittsalters. Auch eine Erhöhung des effektiven Renteneintrittsalters (REA) hat ebenfalls eine relativ große Wirkung auf das Arbeitsangebot, siehe Abbildung 12. Eine Erhöhung um ein Jahr bis 2040 führt zu einer Erhöhung der Erwerbspersonenzahl um 1,2\% bis 2020 und 2,4\% bis 2040. Bei einer Erhöhung um 2 bzw. 3 Jahre steigt die Anzahl der Erwerbspersonen wiederum in etwa proportional. 
ABbILDUNG 12: RENTENEINTRITTSALTER UND ERWERBSPERSONENANZAHL

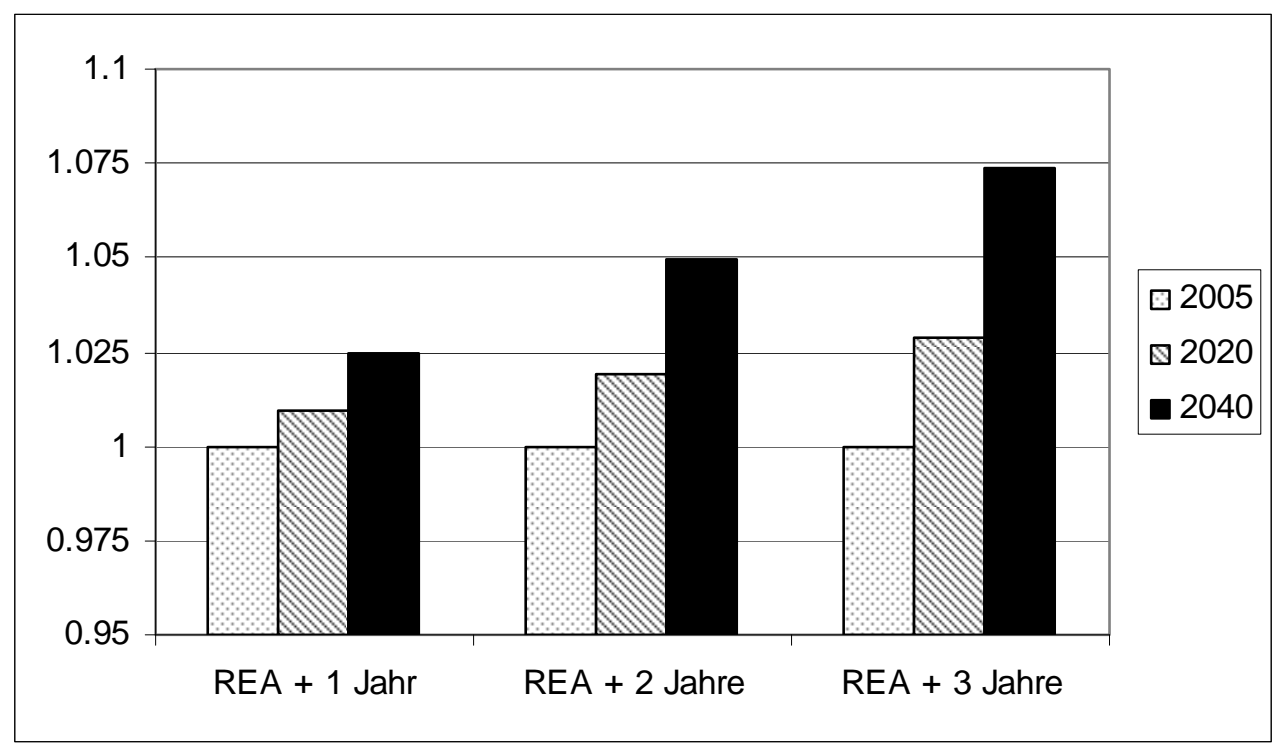

Quelle: MEA: Eigene Berechnungen

Aufgrund der Abschaffung eines Großteils der Frühverrentungswege im Rahmen der 1998er Rentenreform ${ }^{9}$ hat sich das durchschnittliche Renteneintrittsalter für Altersrenten innerhalb der letzten Dekade bereits von 61,2 Jahren in 1995 auf 62,7 Jahren in 2003 erhöht. $^{10}$ Berücksichtigt man Erwerbsminderungsrenten (ab 50 Jahre), so ist das Zugangsalter niedriger (62,3 Jahre in 2005), der Anstieg des effektiven Renteneintrittsalters seit 1996 war aber stärker. ${ }^{11}$ Im Rahmen dieser Entwicklung ist künftig ein weiterer Anstieg um ein Jahr vorstellbar bis die Übergangsregelungen im Jahr 2015 komplett ausgelaufen sind. Eine darüber hinaus gehende Erhöhung des mittleren Renteneintrittsalters um 2 oder gar 3 Jahre baut dann auf der Erhöhung des Regelrentenalters von 65 auf 67 Jahre auf. ${ }^{12}$

Vergleich der Hebelwirkungen. Abbildung 13 zeigt das Wirkungsausmaß der verschiedenen Parameter im Vergleich. ${ }^{13}$ Dabei wird von einer Herabsetzung des Berufseintrittsalters um ein Jahr, einer Anpassung der Frauenerwerbstätigkeit an die Männer um 90\% und einer Erhöhung des Renteneintrittsalters um 2 Jahre ausgegan-

\footnotetext{
${ }^{9}$ Für einen Überblick über den Rentenreformprozess in Deutschland siehe Börsch-Supan und Wilke (2003).

${ }^{10}$ Siehe Berkel und Börsch-Supan (2003). Für eine Diskussion der Wirkung von Frühverrentungsoptionen auf die Wahl des Renteneintrittsalters, siehe auch Schmidt (1995), sowie Riphahn und Schmidt (1997) und Siddiqui (1997).

${ }^{11}$ Siehe Brussig und Wojtkowski (2006).

${ }^{12}$ Siehe hierzu Clemens (2006) und Bucher-Koenen und Wilke (2007).

${ }^{13}$ Üblicherweise werden in der ökonomischen Analyse solche Vergleiche mit Hilfe von Elastizitäten durchgeführt, da so die Wirkungen prozentual normiert werden. In diesem Fall ist dies weniger hilfreich, da nicht klar ist, welche Basis zu verwenden ist. Eine Erhöhung des Renteneintrittsalters um 1 Jahr bspw. auf die gesamte Lebenszeit zu beziehen, erscheint nicht sinnvoll.
} 
gen. Diese Annahmen entsprechen zusammengenommen in etwa dem DänemarkSzenario, werden hier aber einzeln betrachtet.

ABbildung 13: WIRKUNGSAUSMASS DER VERSCHIEDENEN STELLGRÖßEN

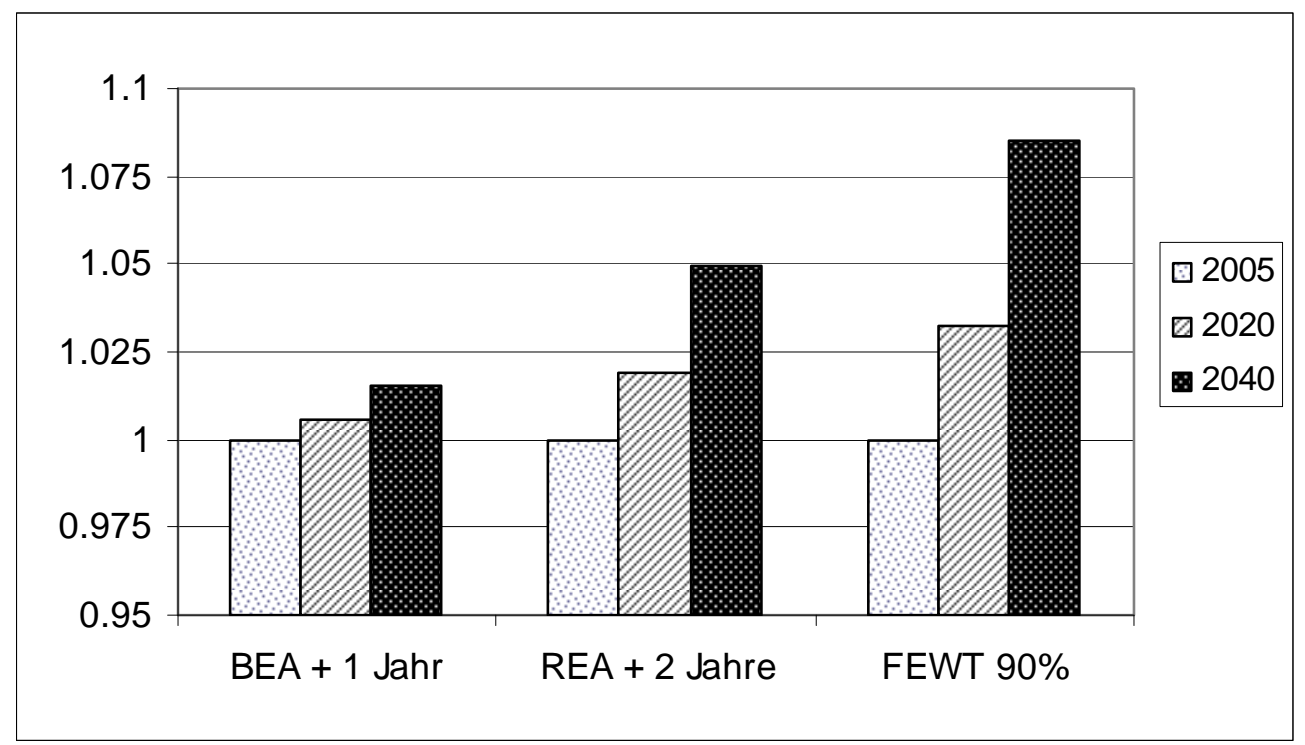

Quelle: MEA: Eigene Berechnungen

Die Herabsetzung des Berufseintrittsalters um ein Jahr hat demnach einen Effekt von 1,6\% mehr Erwerbspersonen in 2040, der Effekt aufgrund der Anpassung der Frauenerwerbstätigkeit beträgt 8,6\% und der Effekt der Anpassung des Renteneintrittsalters 4,9\%. Addiert man diese Teileffekte zusammen, so ergibt sich ein Gesamteffekt von $15,1 \%$ in 2040. Diese Zahl unterschätzt allerdings die vereinte Wirkung der einzelnen Parameterveränderungen, da bspw. eine Erhöhung des mittleren Renteneintrittsalters auch auf den erhöhten Frauenanteil zutrifft, so das der tatsächliche Effekt größer ist (vgl. Abbildung 4). Bei dem Versuch einer Einschätzung der Wirkungsstärke der einzelnen Effekte darf somit nicht übersehen werden, dass die Effekte sich gegenseitig noch verstärken. Die Partialanalyse in diesem Abschnitt darf somit lediglich als Richtungsweiser für die Stärke der einzelnen Effekte gesehen werden.

\section{Projektion der Anzahl der Erwerbstätigen}

Die im vorigen Abschnitt vorgestellten Erwerbsprojektionen geben einen Eindruck darüber, wie groß der demographische Druck auf den Arbeitsmarkt ist und inwieweit Veränderungen im Erwerbsverhalten der Bevölkerung diesem Druck entgegensteuern können. Um die Folgen für die gesamtwirtschaftliche Entwicklung, insbesondere das Wachstum, die Staatsfinanzen und die Entwicklung der sozialen Sicherungssysteme abschätzen zu können, ist eine Projektion der Erwerbspersonen jedoch nicht 
ausreichend. Vielmehr bedarf es zusätzlicher Projektionen der Anzahl der Erwerbstätigen und der Anzahl der Arbeitslosen, da nur die Erwerbstätigen zur Produktion und zu den meisten Steuern beitragen, während die Sozialbeiträge der Arbeitslosen indirekt über die Steuern und Beiträge der Erwerbstätigen finanziert werden müssen.

Im folgenden Abschnitt stellen wir unseren mittel- und langfristig orientierten Ansatz zur Modellierung der Erwerbstätigkeit und der Arbeitslosigkeit dar. Seine konkrete Umsetzung bezüglich unserer vier ausgewählten Szenarien („Status Quo“, „Dänemark“, „Agenda1005“ und „Best Case“) wird in Abschnitt 4.2 beschrieben. Anschließend präsentieren wir die Ergebnisse unserer Erwerbstätigkeitsprojektionen.

\subsection{Modellierung von Arbeitsangebot und Arbeitsnachfrage}

Arbeitsangebot. Mit dem demographischen Wandel sinkt die Anzahl der Personen im erwerbsfähigen Alter, das so genannte Erwerbspersonenpotential. Inwieweit sich diese Entwicklung auf die tatsächliche Anzahl der Erwerbspersonen (Erwerbstätige + Arbeitslose) und damit das Arbeitsangebot auswirkt, hängt davon ab, wie gut das Erwerbspersonenpotential ausgeschöpft werden kann, d.h. wie viele Personen der „stillen Reserve“ (der Differenz zwischen Erwerbspersonenpotential und Erwerbspersonen) tatsächlich Arbeit anbieten. Zur stillen Reserve zählen auch Ausländer, die in den deutschen Arbeitsmarkt eintreten wollen. Sie sind implizit in unseren Wanderungsannahmen enthalten. Damit wird das Arbeitsangebot durch die in Abschnitt 3 vorgestellten Projektionen vollständig dargestellt.

Arbeitsnachfrage. Nicht alle Erwerbspersonen finden jedoch Arbeit. Für die langfristige Entwicklung der Arbeitsnachfrage spielen die Kapital- und Arbeitskosten (Löhne, Lohnnebenkosten und Steuern, vor allem im internationalen Vergleich) und strukturelle Eigenschaften des Arbeitsmarktes (die sich u.a. in Einstellungs- und Kündigungskosten niederschlagen) die entscheidende Rolle, für die kurzfristige Nachfrage auch die Konjunktur.

Modellierungsansatz. In diesem Papier wird bewusst von einer strukturellen Modellierung der Arbeitsnachfrage abgesehen. ${ }^{14}$ Zum einen wollen wir uns auf mittelund langfristige Trends konzentrieren und ignorieren daher Schwankungen über den konjunkturellen Verlauf. Unsere Projektionen stellen also langfristig gleitende Mittelwerte über die Konjunkturzyklen dar. Zum zweiten modellieren wir die konjunkturunabhängige, langfristige Arbeitslosigkeit entsprechend unserer Vorgehensweise in Abschnitt 3 als Szenarien, die sich aufgrund struktureller Parameter, insbesondere

\footnotetext{
${ }^{14}$ Für eine aktuelle Projektion des Arbeitskräftebedarfs bis 2020 siehe Schnur und Zika (2005).
} 
der Arbeitsmarktpolitik, ergeben. Die Anzahl der Erwerbstätigen ergibt sich dann als Differenz: Anzahl der Erwerbspersonen minus Anzahl der Arbeitslosen. Wir treffen implizit somit folgende Annahmen:

1. Veränderungen der Arbeitsnachfrage wirken ausschließlich über die Arbeitslosenquote (ALQ). Wir geben diese szenarisch vor.

2. Änderungen im Arbeitsangebotsverhalten beeinflussen die Arbeitslosenquote nicht, wohl aber die Arbeitslosenzahl: Jedes zusätzliche Arbeitsangebot wird vom Arbeitsmarkt im Umfang von (1-ALQ) absorbiert.

Dieses Verfahren ermöglicht es uns, die sozial- und wirtschaftspolitischen Konsequenzen aus einem künftigen Rückgang in der Erwerbstätigkeit im mittel- und langfristigen Durchschnitt („konjunkturell geglättet“) darzustellen.

\subsection{Szenarien}

Die Szenarien zur Entwicklung der Arbeitslosigkeit werden in Anlehnung an die Annahmen zu den vier Szenarien in Abschnitt 3.2 getroffen. Basisparameter ist die Arbeitslosenquote in der Definition der Bundesagentur für Arbeit, die sich auf alle zivilen Erwerbspersonen bezieht: Anteil der arbeitslos Gemeldeten an den zivilen Erwerbspersonen (Erwerbstätige plus Arbeitslose). Für das Basisjahr 2005 ergibt sich hiernach eine Arbeitslosenquote von etwa 9\%.

Szenario “Status Quo”. Im Szenario „Status Quo“ gehen wir logischerweise davon aus, dass es trotz des Rückgangs in der Anzahl der Erwerbspersonen zu keiner Verringerung der konjunkturbereinigten Arbeitslosigkeit kommt.

Szenario „Agenda 1005“. Unsere Annahmen im Szenario „Agenda1005“ sind ebenfalls vergleichsweise pessimistisch, da die Agenda2010 hauptsächlich an den strukturellen Gründen für die seit den 80er Jahren beharrliche Arbeitslosigkeit ansetzte. Eine nur unvollständige Umsetzung der Agenda2010 wird sich somit in einer nur geringen Änderung der strukturellen Arbeitslosigkeit niederschlagen. Konkret nehmen wir einen langfristigen Rückgang der konjunkturbereinigten Arbeitslosenquote von derzeit 9\% auf 7\% im Jahr 2040 an.

Szenario “Dänemark”. Das Dänemark-Szenario ist mit einer allmählichen Reduktion der Arbeitslosenquote auf das heutige dänische Niveau von 4,8\% kompatibel. ${ }^{15}$ Für unsere Projektionen nehmen wir an, dass dieses Niveau in Deutschland im Jahre

\footnotetext{
${ }^{15}$ Siehe www.statbank.dk.
} 
2040 erreicht wird. In Dänemark konnte eine vergleichbare Reduktion in etwa 10 Jahren erreicht werden. Unser Szenario ist daher recht verhalten optimistisch.

Szenario “Best Case”. Im Best-Case-Szenario gehen wir von einer Absenkung der konjunkturbereinigten Arbeitslosenquote auf das so genannte „natürliche Niveau“ aus, das wir im Einklang mit der Literatur bei 4\% ansetzen. ${ }^{16}$ Tabelle 2 stellt die Szenarien zur Arbeitslosigkeit im Überblick dar:

TABelle 2: Szenarien zur Entwicklung Der Arbeitslosigkeit

\begin{tabular}{lll}
\hline Szenario & $\mathbf{2 0 0 5}$ & $\mathbf{2 0 4 0}$ \\
\hline “Status Quo” & $9,1 \%$ & $9,1 \%$ \\
“Agenda 1005” & $9,1 \%$ & $7,0 \%$ \\
“Dänemark” & $9,1 \%$ & $4,8 \%$ \\
“Best Case” & $9,1 \%$ & $4,0 \%$ \\
\hline
\end{tabular}

Die Übergänge in den letzten drei Szenarien finden wie in Abschnitt 3.2 beschrieben linear zwischen den Jahren 2008 und 2040 statt. Nach 2040 halten wir die Arbeitslosenquote konstant. Technisch nehmen wir in den Übergangsszenarien an, dass ein den Szenarien entsprechender Anteil der in der Vorperiode arbeitslosen Personen in der Folgeperiode den Sprung in die Erwerbstätigkeit schafft. Zudem wird von einer konstanten Altersverteilung der Arbeitslosen gemäß Abbildung 3 sowie einer über alle Altersjahre gleichmäßigen Reduktion der Arbeitslosigkeit ausgegangen.

\footnotetext{
${ }^{16}$ Nach der Theorie von Milton Friedman (1968) schwankt die Arbeitslosenquote einer Volkswirtschaft langfristig um eine natürliche, positive Arbeitslosenquote. In der Literatur wird diese in der Regel bei $4 \%$ angenommen.
} 
Abbildung 14: Alters- und geschlechtsspezifische ArbeitslosenQUOTEN

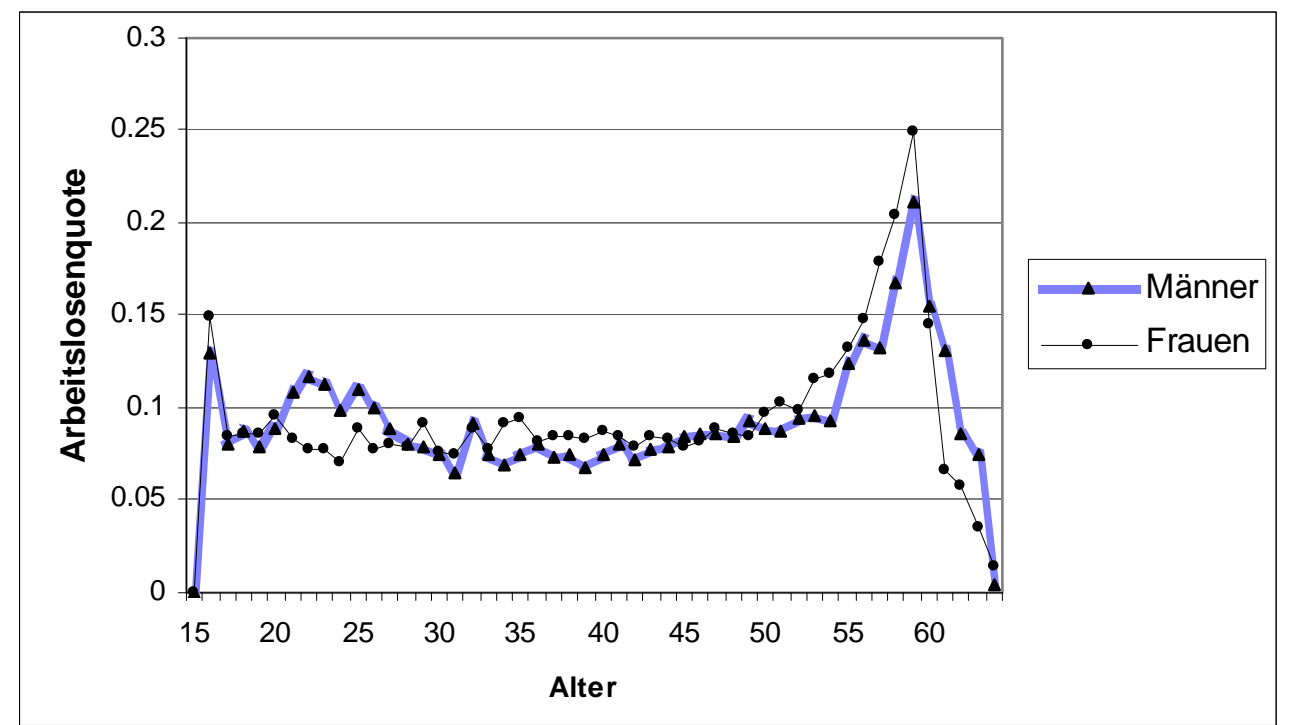

Quelle: Eigene Berechnungen auf Basis von Daten des Statistischen Bundesamtes (www.destatis.de).

\subsection{Ergebnisse}

Im Folgenden zeigen wir zunächst die künftige Entwicklung der Erwerbstätigenanzahl auf und vergleichen diese mit unseren in Abschnitt 3.3 präsentierten Erwerbspersonenzahlen. Danach betrachten wir die Entwicklung der relativen Erwerbstätigenanzahl (im Verhältnis zur Gesamtbevölkerung). Schließlich setzen wir die Anzahl der Erwerbstätigen ins Verhältnis zur Anzahl der Rentner und betrachten die künftige Entwicklung des Rentnerquotienten.

Entwicklung der Anzahl der Erwerbstätigen. Ebenso wie die Anzahl der Erwerbspersonen geht die Anzahl der Erwerbstätigen in allen vier Szenarien zurück (siehe Abbildung 15). Im Dänemark-Szenario reduziert sich die Erwerbstätigenanzahl bis 2040 um über 2,5 Mio. auf etwa 36,2 Mio. Im Szenario „Best Case“ sind es 37,6 Mio, im Szenario Agenda 1005 nur noch 33,7 Mio. in 2040. Im Status Quo ergibt sich ein Rückgang von über 11 Mio. auf knappe 27,4 Mio. Erwerbstätige in 2040. Letzteres entspricht einem Rückgang von fast 30\%.

Die Differenz zwischen der Erwerbspersonen- und der Erwerbstätigenanzahl verringert sich dabei von etwa 3,5 Mio. heute auf etwa 2,8 Mio. im Jahr 2040 im Status Quo, 2,5 Mio. im Szenario Agenda 1005 und etwa 1,7 Mio. in den Szenarien „Dänemark“ und „Best Case“. Dies ist erstens darauf zurückzuführen, dass sich die Arbeitslosenquote im Zeitverlauf auf eine immer geringere Erwerbspersonenanzahl bezieht. Zweitens verringert sich die Differenz im Dänemark- und Best-CaseSzenario zusätzlich aufgrund der annahmegemäßen Reduktion der Arbeitslosigkeit. 
ABbildung 15: ENTWICKLUNG DER ERWERBSPERSONEN- SOWIE ERWERBSTÄTIGENANZAHL

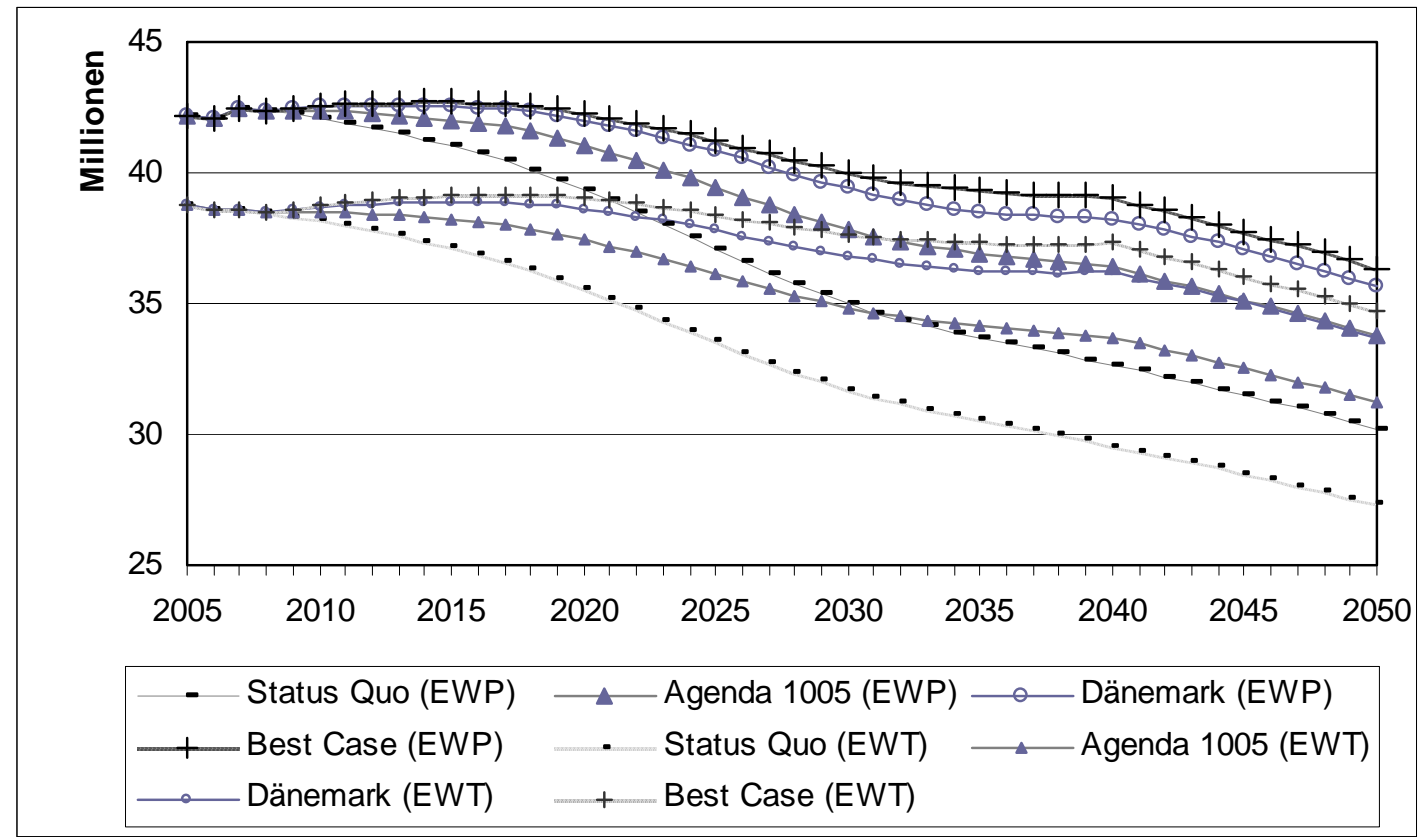

Quelle: MEA: Eigene Berechnungen

Ökonomische Stützquote. Nicht nur die Anzahl der Erwerbstätigen, auch die Bevölkerung geht zurück. Der relative Rückgang in der Anzahl der Erwerbstätigen fällt somit geringer aus als der absolute Rückgang. Dies wird in Abbildung 16 anhand der Stützquote veranschaulicht. Die Stützquote ist hier definiert als der Anteil der Erwerbstätigen an der erwachsenen Bevölkerung ab dem Alter 15. Dies entspricht im Großen und Ganzen der Anzahl der Konsumenten, deren Nachfrage nach Gütern und Dienstleistungen von den Erwerbstätigen produziert wird. Die Stützquote beschreibt also, worauf sich der Konsum einer Gesellschaft am Arbeitsmarkt stützen kann. 
Abbildung 16: EnTwicklung Der Stützquote

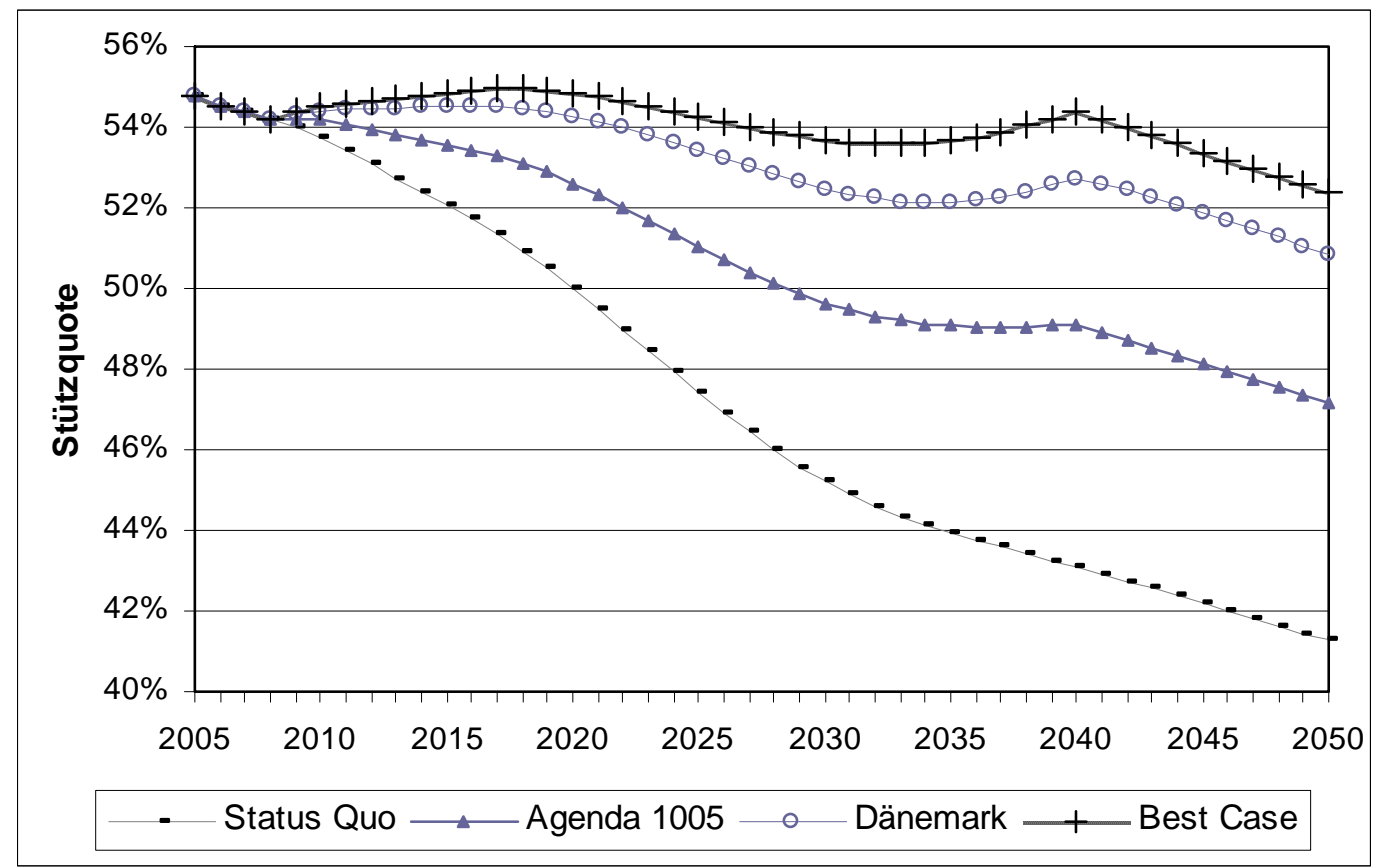

Quelle: MEA: Eigene Berechnungen

Mit Ausnahme des Status-Quo-Szenarios beginnt der wesentliche Rückgang hier erst ab 2015. Für das Dänemark-Szenario fällt die Stützquote von heute 54,8\% auf 52,1\% in 2035. Danach steigt die Stützquote bis 2040 wieder an. Der erneute Anstieg hängt mit den unterstellten Anpassungen am Arbeitsmarkt zusammen, die aufgrund der Alterstruktur die Anzahl der Erwerbstätigen nach 2035 schneller wachsen lassen als die Zunahme in der Lebenserwartung die erwachsene Gesamtbevölkerung wachsen lässt. Nach 2040 geht die Stützquote bei konstanten Erwerbstätigenquoten und sinkenden Bevölkerungszahlen weiter zurück. Für das Status-Quo-Szenario, in dem keine Anpassungen am Arbeitsmarkt angenommen werden, ist die Stützquote im gesamten Zeitraum steil und streng monoton fallend.

Rentnerquotient. Noch gravierender als der Rückgang in der absoluten bzw. relativen Anzahl der Erwerbstätigen ist die Veränderung des Verhältnisses von Erwerbstätigen zu Rentnern, dem sogenannten Rentnerquotienten. Dieser beschreibt die Anzahl der Rentner im Verhältnis zur Anzahl der Erwerbstätigen. ${ }^{17}$

Abbildung 17 zeigt den Rentnerquotienten für die verschiedenen Arbeitsmarktszenarien.

\footnotetext{
${ }^{17}$ Als Rentner betrachten wir in diesem Papier generell alle aus dem Arbeitsmarkt ausgeschiedenen Personen, als Erwerbstätige zählen Arbeitnehmer und Selbstständige. Der Rentnerquotient fällt hier somit niedriger aus als in der klassischen Betrachtung der Rentenversicherung, bei der die eine staatliche Rente beziehenden Personen ins Verhältnis zu den Beitragszahlern gesetzt werden.
} 


\section{Abbildung 17: ENTWiCKLUng Des Rentnerquotienten}

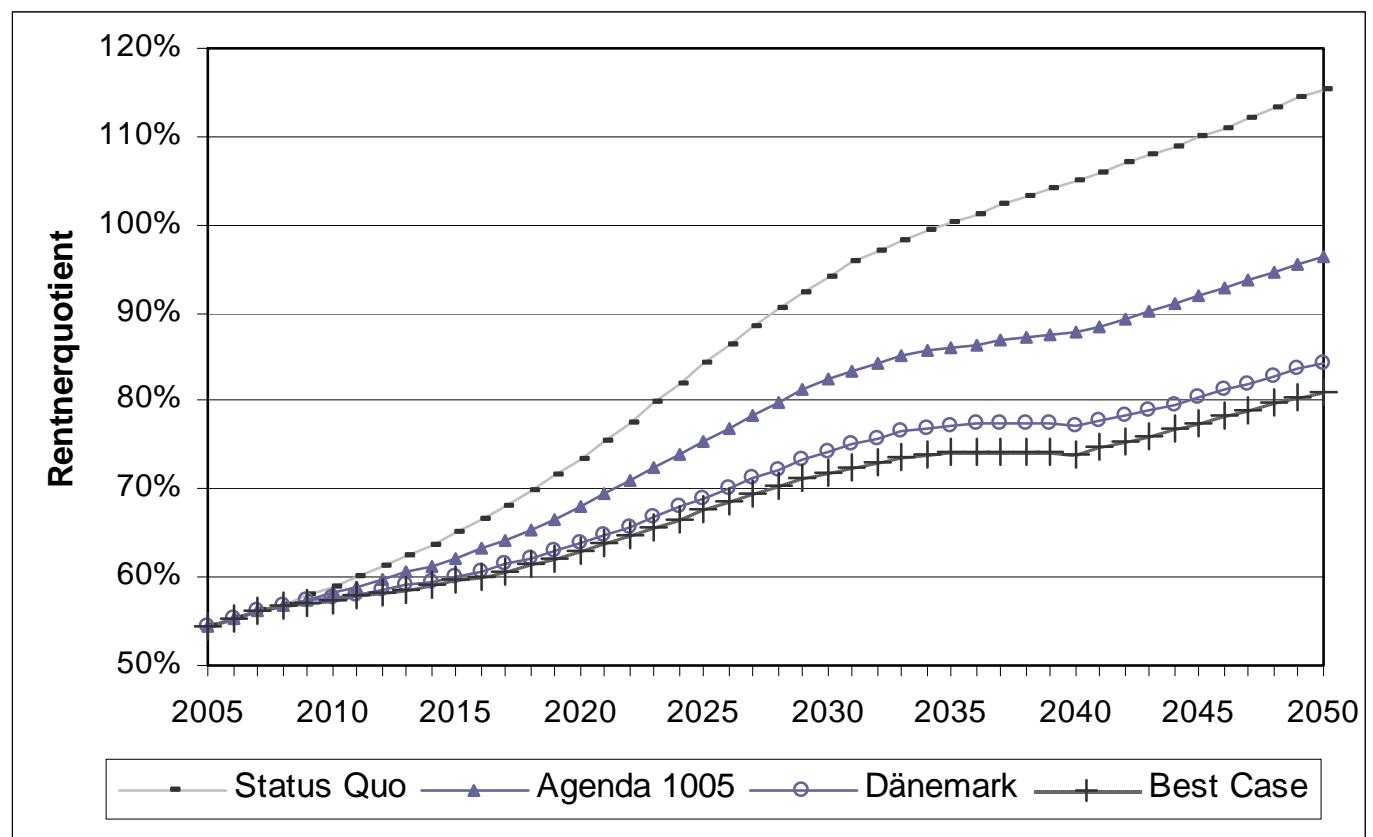

Quelle: MEA: Eigene Berechnungen

In allen Szenarien kommt es zu einem Anstieg des Rentnerquotienten. Die Spannweite zwischen den beiden Extremszenarien ist allerdings groß. Bei einer sehr positiven Erwerbstätigkeitsentwicklung wie im Best-Case-Szenario steigt der Rentnerquotient bis auf etwa 73,1\% in 2040 an. Im Status Quo hingegen erhöht sich der Rentnerquotient bis zum Jahr 2040 auf knapp 105\%, d.h. dass 2040 in diesem Fall ein Erwerbstätiger alleine mehr als einen Rentner finanzieren muss. Im DänemarkSzenario ist der Rentnerquotient 2040 um etwa 4 Prozentpunkte höher als im BestCase-Szenario. Auch hier zeigt sich wieder, dass selbst bei einer nahezu bestmöglichen Ausschöpfung des Erwerbspersonenpotentials der langfristige Trend nicht komplett umgangen werden kann, dass jedoch der unvermeidliche Anstieg auf einem deutlich geringeren Niveau stattfindet.

Konsequenzen eines stärkeren Bevölkerungsrückgangs. Wie sehr basiert dieser Anstieg des Rentnerquotienten auf der zugrunde liegenden Bevölkerungsprojektion? Abbildung 18 zeigt die Entwicklung des Rentnerquotienten im Dänemark-Szenario für die beiden MEA-Bevölkerungsprojektionen sowie für die Variante 1W1 der 11. Koordinierten Bevölkerungsvorausberechnung. 
AbBildung 18: ENTWiCKLUng Des Rentnerquotienten Bei ALTERNATIVEN BEVÖLKERUNGSPROGNOSEN

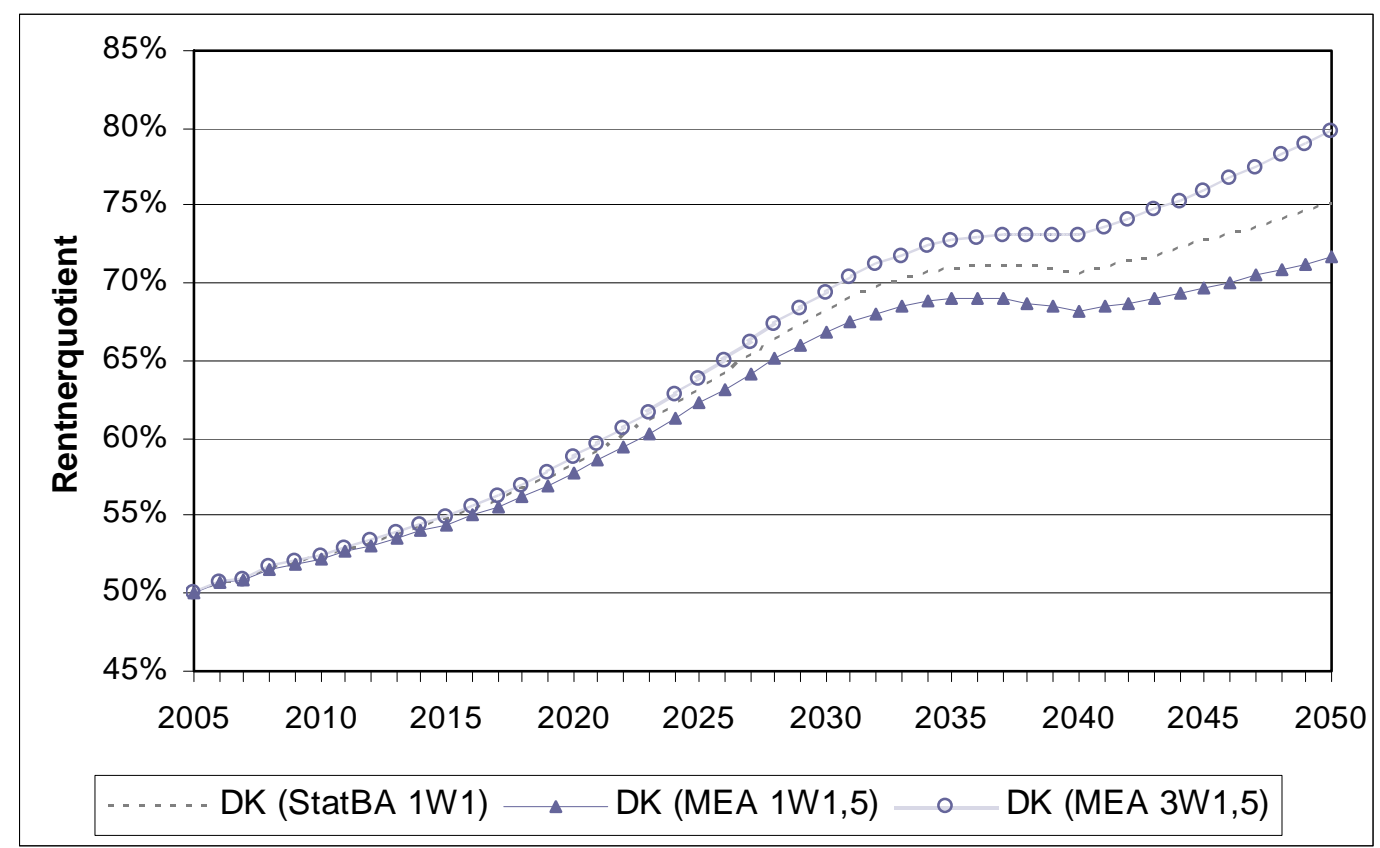

Quelle: MEA: Eigene Berechnungen

Werden pessimistischere Annahmen an die Lebenserwartung getroffen (MEA 1W1,5), so fällt der Rentnerquotient geringer aus, da eine geringere Anzahl an Rentnern einer nahezu unveränderten Anzahl an Erwerbstätigen (vgl. Abbildung 8) gegenüber steht. Unterstellt man eine geringere Migration wie bspw. im Szenario 1W1 des Statistischen Bundesamtes, so liegt der Anstieg des Rentnerquotienten für alle drei Arbeitsmarktszenarien graphisch jeweils etwa zwischen den Verläufen in den beiden MEA-Bevölkerungsprojektionen. Der aufgrund der moderateren Annahmen an die Lebenserwartung tendenziell geringere Anstieg des Rentnerquotienten wird hier wegen des geringeren Wanderungssaldos und der allgemein jüngeren Altersstruktur der Migranten teils kompensiert.

Der Druck auf die sozialen Sicherungssysteme kann somit nur teilweise über den Arbeitsmarkt abgefedert werden. Vor allem dem Anstieg in der Lebenserwartung kann nur sehr begrenzt eine höhere Erwerbsbeteiligung Älterer entgegengesetzt werden. Selbst bei äußerst pessimistischen Annahmen über die künftige Entwicklung der Lebenserwartung bleibt der Druck groß. Es ist davon auszugehen, dass der Rentnerquotient innerhalb der nächsten dreißig Jahre auch im bestmöglichen Fall (Szenario „Best Case“ basierend auf MEA 1W1,5) um mindestens 15 Prozentpunkte ansteigen wird. Dies bedeutet für die aktive erwerbstätige Bevölkerung eine zusätzliche Belastung allein bis 2040 von mehr als einem Viertel. 


\section{Fazit und Ausblick}

Dieser Beitrag zeigt auf, welche künftige Arbeitsangebots- und Erwerbstätigenentwicklung für Deutschland bis 2040 bzw. 2050 erwartet werden kann. Es wurde deutlich, dass diese Entwicklung maßgeblich davon abhängt, wie sehr es gelingt, durch Arbeitsmarktreformen entsprechende Veränderungen des Arbeitsangebotsverhaltens und der Arbeitsnachfrage einzuleiten.

Spätestens nach 2020 wird sowohl die Anzahl der Erwerbspersonen als auch die Anzahl der Erwerbstätigen aufgrund des Bevölkerungsrückgangs spürbar zurückgehen. Weder eine bestmögliche Ausnutzung des Erwerbspersonenpotenzials noch eine realistische Zunahme der Migration können diesen Trend vollständig verhindern.

Im Dänemark-Szenario geht die Anzahl an Erwerbspersonen etwa ab 2015 langsam zurück. Den gleichen Trend für Deutschland findet auch das Institut für Arbeitsmarkt- und Berufsforschung (IAB) in seinen Arbeitsmarktprojektionen und Langfristberechnungen zum Erwerbspersonenpotenzial ${ }^{18}$, sowie die Europäische Kommission $^{19}$, obwohl sich die Prognosen im Detail aufgrund unterschiedlicher Bevölkerungs- und Erwerbstätigkeitsannahmen unterscheiden. Eine wichtige Einsicht ist, dass sich das Niveau dieses Rückgangs in den Arbeitsmarktreformszenarien deutlich im Vergleich zum Status Quo mindern lässt.

Auch das Durchschnittsalter der Erwerbspersonen wird in den kommenden Dekaden steigen. Insbesondere wird der Anteil der Erwerbspersonen im Alter 55+ deutlich zunehmen. Diese Veränderung in der Altersstruktur ist unumgänglich und erfordert vor allem entsprechende Anpassungsprozesse seitens der Arbeitgeber und -nehmer.

Ein Anstieg des Rentnerquotienten ist daher angesichts des starken demographischen Wandels unaufhaltbar. Auch hier gilt jedoch, dass das Ausmaß des Anstiegs wesentlich von den Veränderungen im Erwerbsverhalten der Bevölkerung abhängt.

Ein Großteil des demographischen Drucks kann also durch den Arbeitsmarkt abgefedert werden. Hierzu sind gezielte Reformen notwendig, um die notwendigen Anreize für eine höhere Erwerbsbeteiligung zu schaffen.

Übereinstimmung mit anderen Prognosen. Ein Vergleich der sich im DänemarkSzenario ergebenden absoluten Erwerbstätigenzahlen und des Rentnerquotienten mit der Erwerbstätigenprognose der Rürup-Kommission ${ }^{20}$ zeigt eine große Übereinstimmung beider Prognosen. Dies ist insofern überraschend, als die Erwerbstätigenprog-

\footnotetext{
${ }^{18}$ Siehe Fuchs und Dörfler (2005 a und 2005b), sowie Fuchs und Söhnlein (2007).

${ }^{19}$ Siehe Carone (2005).

${ }^{20}$ Vgl. Kommission für die Nachhaltigkeit in der Finanzierung der Sozialen Sicherungssysteme (2003).
} 
nose der Kommission erstens auf einer bis 2050 wesentlich optimistischeren Bevölkerungsprognose (vgl. „Rürup“ in Abbildung 1) basiert, und sie zweitens neben dem Arbeitsangebot auch explizit die Entwicklung der künftigen Arbeitsnachfrage berücksichtigt, wovon in diesem Papier abgesehen wurde. Diese Koinzidenz veranschaulicht, wie komplex der Einfluss unterschiedlicher Prognoseannahmen auf die Ergebnisse ist, und dass abweichende Bevölkerungs- und Arbeitsmarktannahmen sich in ihrer Wirkung gegenseitig aufheben können.

Vor allem zeigt sich, dass innerhalb einer realistischen Szenarienspannbreite die Ergebnisse recht robust sind: Welche künftige Entwicklung auch immer letztlich realisiert wird, sie kann keine Überraschung sein, denn die natürlichen Ober- und Untergrenzen sind bekannt („Status Quo“ und „Best Case“).

Erforderlicher Produktivitätszuwachs. Wie wir gesehen haben, wird nicht nur absolut, sondern auch relativ zur Bevölkerung die Anzahl der Erwerbstätigen sinken. Relativ zum bislang gewohnten Konsum von Gütern und Dienstleistungen und den entsprechenden Investitionen wird es weniger Erwerbstätige geben, die diese produzieren können. Mit einer geringeren Produktion können wir jedoch nicht ohne Weiteres unseren gewohnten Lebensstandard halten. Um wie gewohnt weiter zu konsumieren und zu investieren, müsste Deutschland daher in Zukunft einen größeren Produktivitätszuwachs verzeichnen als bisher.

Konkret führt der relative Rückgang in der Anzahl der Erwerbstätigen allein bis 2035 dazu, dass im Dänemark-Szenario die Erwerbstätigen im Jahre 2035 nahezu 5\% mehr leisten müssten als heute, um die gleiche Menge an Konsum- und Investitionsgütern pro Kopf zu produzieren. Dies entspräche einem jährlichen Produktivitätszuwachs von etwa 0,2 Prozentpunkten bis 2035, zusätzlich zum historische normalen Produktivitätszuwachs von etwa $1,4 \%{ }^{21}$ pro Jahr. Ein jährlicher Produktivitätszuwachs von $1,6 \%$ ist nicht unmöglich in vielen Ländern sogar normal; er würde den Lebensstandard relativ zu unseren EU-Nachbarn in etwa stabil halten.

Im Status quo Szenario müssten die Erwerbstätigen im Jahr 2035 hingegen schon fast 20\% mehr leisten als heute. Dies entspräche einem jährlichen Produktivitätszuwachs von etwa 0,63 Prozentpunkten bis 2035. Eine Steigerung des heutigen Produktivitätsfortschritts auf über 2\% pro Jahr für die gesamte mittelfristige Zukunft ist nur schwer vorstellbar. Das Status-Quo-Szenario impliziert daher - ohne einen historisch gesehen erstaunlichen Sprung in der Produktivität - eine Einbuße unseres gewohnten Lebensstandards relativ zu unseren Nachbarn.

\footnotetext{
${ }^{21}$ Siehe Buchheim (1998). Das Institut für Arbeitsmarkt- und Berufsforschung (IAB) rechnet in seiner Langfristprojektion des Arbeitskräftebedarfs bis 2020 sogar nur mit 1,3\%, siehe Schnur und Zika (2005).
} 
Unsere Projektionen zeigen also, wie wichtig derzeitige und künftige Arbeitsmarktreformen sind, um auch in Zukunft unseren Lebensstandard, jedenfalls relativ zu unseren Nachbarländern, aufrechterhalten zu können.

Wirtschaftspolitische Implikationen. Welche Konsequenzen für die Wirtschaftspolitik lassen sich daraus ziehen? Diese Frage soll und kann im Rahmen dieses eher technischen Papiers nicht umfassend beantwortet werden. Einige wesentliche Aspekte sollen hier jedoch abschließend kurz angeführt werden.

Abschnitt 3.5 hat gezeigt, dass neben kürzeren Ausbildungszeiten und einem damit einhergehenden niedrigeren mittleren Berufseintrittsalter insbesondere eine Erhöhung der Frauenerwerbstätigkeit bzw. eine Heraufsetzung des Renteneintrittsalters große Auswirkungen auf das Arbeitsangebot haben. In Bezug auf die Frauenerwerbstätigkeit muss dazu in Deutschland vor allem eine bessere Vereinbarkeit von Familie und Beruf zu schaffen. ${ }^{22}$ So ist die Erwerbsbeteiligung kinderloser Frauen in Deutschland im internationalen Vergleich gut; erst wenn Mütter mit Kindern betrachtet werden, fällt Deutschland im Ranking zurück. ${ }^{23}$

Eine Heraufsetzung des Renteneintrittsalters wirkt sich nicht nur positiv auf die Anzahl der Erwerbspersonen, sondern ebenso günstig auf die Anzahl der Rentner aus und trägt so zu einem geringeren Anstieg des Rentnerquotienten bei. Neben einem aufgrund der vergangenen Rentenreformen 1992, 2001 und 2004 zu erwartenden weiteren Anstieg des mittleren Renteneintrittsalter lässt sich ein weiterer Anstieg durch eine Anhebung des gesetzlichen Rentenalters auf 67 Jahre erreichen.

Schließlich ist das Arbeitsvolumen insgesamt, also das die tatsächlich geleistete Arbeitszeit der Erwerbspersonen (in h), von großer Bedeutung. Der Beschäftigungszuwachs der Frauen in den 90er Jahren in Deutschland basiert bspw. nahezu vollständig auf Teilzeitarbeitzuwächsen. ${ }^{24}$ Insgesamt lässt sich feststellen, dass das Arbeitsvolumen aufgrund der geringeren Wochenarbeitszeit, vermehrten Urlaubsansprüchen, der im Vergleich zu früher geringen Erwerbsbeteiligung der Älteren und nicht zuletzt der hohen Arbeitslosigkeit in den vergangenen Dekaden stark zurückgegangen ist. ${ }^{25}$ Es gibt jedoch einige Indizien dafür, dass das Arbeitsvolumen im Zuge der erwarteten Annäherung der Erwerbsmuster von Frauen und Männern künftig wieder etwas ansteigen wird. ${ }^{26}$ So lassen sich bspw. im europäischen Vergleich umso niedrigere Arbeitszeitdifferenzen zwischen Männern und Frauen ausmachen, je besser die vor-

\footnotetext{
${ }^{22}$ Vgl. Wissenschaftlicher Beirat (2005).

${ }^{23}$ Siehe Dressel, Cornelißen und Wolf (2005).

${ }^{24}$ Siehe Bothfelt, Klammer und Klenner (2005), sowie Dressel (2005).

${ }^{25}$ Siehe Börsch-Supan (2000).

${ }^{26}$ Siehe z.B. Allmendinger und Ebner (2005).
} 
handene Infrastruktur von Kindererziehungsleistungen. ${ }^{27}$ Dies würde die finanzielle Lage der Sozialversicherungen zumindest mittelfristig günstig beeinflussen.

Schließlich spielt neben der in diesem Beitrag quantitativ skizzierten Entwicklung auch die qualitative Entwicklung auf dem Arbeitsmarkt eine wichtige Rolle. So kann es aufgrund des zunehmend steigenden Bedarfs an Höherqualifizierten und der zugleich schrumpfenden Anzahl jüngerer Erwerbspersonen bspw. bei einem Stillstand der Bildungsentwicklung zu einem deutlichen Fachkräftemangel kommen. ${ }^{28}$ Gleichzeitig bestehen bereits heute teils massive Beschäftigungsprobleme wettbewerbsschwacher Arbeitnehmer. Hier ist eine entsprechende Bildungs- und Weiterbildungspolitik äußerst wichtig, um einen solchen Mismatch auf dem Arbeitsmarkt zu verhindern. In jedem Fall kommt der Akkumulation von Humanvermögen durch Aus- und Weiterbildung künftig ein immer höherer Stellenwert zu.

Eine aktivierende Arbeitsmarktpolitik im Sinne einer sowohl quantitativen als auch qualitativen Erschließung des Erwerbspersonenpotenzials wird somit künftig immer wichtiger werden. Dieser Beitrag hat gezeigt, dass eine solche Politik die Auswirkungen der Bevölkerungsalterung auf den Arbeitsmarkt deutlich abfedern kann. Auch der Druck auf die sozialen Sicherungssysteme kann durch eine - in diesem Beitrag sehr maßvolle und graduelle - Reformpolitik signifikant abgemildert werden. Ein Ende des derzeitigen Reformprozesses hätte, wie die alternativen Szenarien aufzeigen, dagegen langfristig klar restriktive Folgen für den Lebensstandard und die Nachhaltigkeit unserer Sozialsysteme. Der Unterschied zwischen dem Reformszenario „Dänemark“ und dem Szenario einer nur unvollständig realisierten Agenda2010 („Agenda1005“) beträgt 2.1 Mio. Erwerbstätige im Jahr 2030. Deren Arbeitskraft ist unverzichtbar für unseren Lebensstandard und unsere Sozialversicherungen.

\footnotetext{
${ }^{27}$ Siehe hierzu auch European Commission (2000).

${ }^{28}$ Siehe hierzu Reinberg und Hummel (2003).
} 


\section{Literaturverzeichnis}

Allmendinger, J. und C. Ebner (2005): Demographischer Wandel. Chancengleichheit für Frauen auf dem Arbeitsmarkt - was ist zu tun? In: Demographischer Wandel - Herausforderung und Chance für den Arbeitsmarkt, Dokumentation ESF Kongress Hessen 2005, Wiesbaden

Berkel, B., Börsch-Supan, A. und A. Ludwig (2002): Sind die Probleme der Bevölkerungsalterung durch eine höhere Geburtenrate lösbar? MEA Arbeitspapier 2502, MEA, Universität Mannheim.

Berkel, B. und A. Börsch-Supan (2003): Renteneintrittsentscheidungen in Deutschland: Langfristige Auswirkungen verschiedener Reformoptionen, MEA Arbeitspapier 31-03, MEA, Universität Mannheim.

Börsch-Supan, A. (2002a): Mehr Zuwanderung? Zur Rolle des Auslands bei der Stabilisierung der gesetzlichen Rentenversicherung in Deutschland, MEA Arbeitspapier 22-02, MEA, Universität Mannheim.

Börsch-Supan, A. (2002b): Übergang statt Untergang: Wider die Furcht vor dem Untergang der Arbeit, MEA Arbeitspapier 04-02, MEA, Universität Mannheim.

Börsch-Supan, A. (2003): Labour Market Effects of Population Aging, Review of Labour Economics and Industrial Relations 17, Special Issue 2003, 5-44.

Börsch-Supan, A., und C. Wilke (2003): The German Social Security System: How it Was and How it Will Be, MRRC Discussion Paper UM03-01, Michigan Retirement Research Center, University of Michigan.

Bothfeld, S., Klammer, U. und C. Klenner (2005): WSI-FrauenDatenReport 2005, Handbuch zur wirtschaftlichen und sozialen Situation von Frauen, Edition Sigma, Dezember 2005.

Brussig, M. und S. Wojtkowski (2006): Durchschnittliches Renteneintrittsalter steigt weiter, Altersübergangsreport 2006-02, Hans-Böckler-Stiftung, Düsseldorf und Institut Arbeit und Technik, Gelsenkirchen.

Bucher-Koenen, T. und C. Wilke (2007): Langfristige Auswirkungen der Altersgrenzenanhebung, MEA Arbeitspapier 2007, MEA, Universität Mannheim, im Erscheinen.

Buchheim, C. (1994), Industrielle Revolutionen, dtv: München.

Carone, G. (2005): Long-term labour force projections fort he 25 EU Member States: A set of data for assessing the economics impact of ageing, European Commission Economic Papers, Nr. 235/ November 2005.

Clemens, J. (2006): Anmerkungen zur geplanten Anhebung des gesetzlichen Rentenalters, Wirtschaftsdienst, Nr. 3/2006.

Dressel, C. (2005): Erwerbstätigkeit - Arbeitsmarktintegration von Frauen und Männern, In: Bundesministerium für Familie, Senioren, Frauen und Jugend (Hrsg.): 1. Datenreport zur Gleichstellung von Frauen und Männern in der Bundesrepublik Deutschland, Berlin. 
Dressel, C, Cornelißen, W. und K. Wolf (2005): Vereinbarkeit von Familie und Beruf, In: Bundesministerium für Familie, Senioren, Frauen und Jugend (Hrsg.): 1. Datenreport zur Gleichstellung von Frauen und Männern in der Bundesrepublik Deutschland, Berlin.

European Commission (2000): Employment in Europe 2000. Luxemburg.

Friedman, M. (1968): The role of monetary policy, American Economic Review, Vol. 58 (May), 1-17.

Fuchs, J. und K. Dörfler (2005a): Projektion des Erwerbspersonenpotenzials bis 2050 - Annahmen und Datengrundlage, IAB Forschungsbericht, Nr. 25/2005.

Fuchs, J. und K. Dörfler (2005b): Projektion des Arbeitsangebots bis 2050 - Demografische Effekte sind nicht mehr zu bremsen, IAB Kurzbericht, Nr.11/2005.

Fuchs, J. und D. Söhnlein (2007): Einflussfaktoren auf das Erwerbspersonenpotenzial - Demografie und Erwerbsverhalten in Ost- und Westdeutschland, IAB Discussion Paper, Nr. 12/2007.

Kommission für die Nachhaltigkeit in der Finanzierung der Sozialen Sicherungssysteme (2003): Nachhaltigkeit in der Finanzierung der Sozialen Sicherungssysteme, Bericht der Kommission, Bundesministerium für Gesundheit und Soziale Sicherung, Berlin.

Reinberg, A. und M. Hummel (2003): Bildungspolitik: Steuert Deutschland langfristig auf einen Fachkräftemangel hin? IAB Kurzbericht, Nr. 9/2003.

Riphahn, R.T., and P. Schmidt (1997), Determinanten des Ruhestandes: Lockt der Ruhestand oder drängt der Arbeitsmarkt? Jahrbücher für Wirtschaftswissenschaften, 48(1), 113-147.

Schmidt, P. (1995), Die Wahl des Rentenalters - Theoretische und empirische Analyse des Rentenzugangsverhaltens in West- und Ostdeutschland, Lang: Frankfurt.

Schnabel, S., Kistowski, K. und J. Vaupel (2005): Immer neue Rekorde und kein Ende in Sicht, in: J.M. Hoern und J.W. Vaupel (2005): Demografische Forschung - Aus Erster Hand, Jg.2, Nr.2, Max-Planck-Institut für demographische Forschung, Rostock.

Schnur, P. und G. Zika (2005): Projektion des Arbeitskräftebedarfs bis 2020 - Nur zögerliche Besserung am deutschen Arbeitsmarkt, IAB Kurzbericht, Nr. 12/2005.

Siddiqui, S. (1997), The Pension Incentive to Retire: Empirical Evidence for West Germany, Journal of Population Economics 10(4), 463-86.

Statistisches Bundesamt (2006): 11. koordinierte Bevölkerungsvorausberechnung Annahmen und Ergebnisse, Statistisches Bundesamt, Wiesbaden, www.destatis.de

Wissenschaftlicher Beirat beim Bundesministerium für Wirtschaft und Arbeit (2005): Alterung und Familienpolitik, Bundesministerium für Wirtschaft und Arbeit, Berlin. 


\section{Discussion Paper Series}

Mannheim Research Institute for the Economics of Aging Universität Mannheim

To order copies, please direct your request to the author of the title in question.

\begin{tabular}{|c|c|c|c|}
\hline Nr. & Autoren & Titel & Jahr \\
\hline $141-07$ & Hendrik Jürges & $\begin{array}{l}\text { Healthy minds in healthy bodies. An } \\
\text { international comparison of education-related } \\
\text { inequality in physical health among older adults }\end{array}$ & 07 \\
\hline $142-07$ & $\begin{array}{l}\text { Karsten Hank, } \\
\text { Stephanie Stuck }\end{array}$ & $\begin{array}{l}\text { Volunteer Work, Informal Help, and Care among } \\
\text { the 50+ in Europe: Further Evidence for 'Linked' } \\
\text { Productive Activities at Older Ages }\end{array}$ & 07 \\
\hline $143-07$ & Jürgen Maurer & $\begin{array}{l}\text { Assessing Horizontal Equity in Medication } \\
\text { Treatment Among Elderly Mexicans: Which } \\
\text { Socioeconomic Determinants Matter Most? }\end{array}$ & 07 \\
\hline $144-07$ & Jürgen Maurer & $\begin{array}{l}\text { Socioeconomic and Health Determinants of } \\
\text { Health Care Utilization Among Elderly } \\
\text { Europeans: A Semiparametric Assessment of } \\
\text { Equity, Intensity and Responsiveness for Ten } \\
\text { European Countries }\end{array}$ & 07 \\
\hline $145-07$ & Jürgen Maurer & $\begin{array}{l}\text { Modelling socioeconomic and health } \\
\text { determinants of health care use: } A \\
\text { semiparametric approach }\end{array}$ & 07 \\
\hline $146-07$ & $\begin{array}{l}\text { Jérome Adda, James } \\
\text { Banks, Hans-Martin } \\
\text { von Gaudecker }\end{array}$ & $\begin{array}{l}\text { The Impact of Income Shocks on Health: } \\
\text { Evidence from Cohort Data }\end{array}$ & 07 \\
\hline 147-07 & $\begin{array}{l}\text { Klaus Jäger, } \\
\text { Wolfgang Kuhle }\end{array}$ & $\begin{array}{l}\text { The Optimum Growth Rate for Population } \\
\text { Reconsidered }\end{array}$ & 07 \\
\hline $148-07$ & $\begin{array}{l}\text { Axel Börsch-Supan, } \\
\text { Ismail Düzgün, } \\
\text { Matthias Weiss }\end{array}$ & $\begin{array}{l}\text { Age and Productivity in Work Teams: Evidence } \\
\text { from the Assembly Line }\end{array}$ & 07 \\
\hline $149-07$ & Matthias Weiss & Sick Leave and the Composition of Work Teams & 07 \\
\hline $150-07$ & Matthias Weiss & Are Age-Diverse Work Teams Better? & 07 \\
\hline $151-07$ & $\begin{array}{l}\text { Alexander Ludwig, } \\
\text { Thomas Schelkle, } \\
\text { Edgar Vogel }\end{array}$ & $\begin{array}{l}\text { Demographic Change, Human Capital and } \\
\text { Endogenous Growth }\end{array}$ & 07 \\
\hline $152-07$ & $\begin{array}{l}\text { Karsten Hank } \\
\text { Stephanie Stuck }\end{array}$ & $\begin{array}{l}\text { Gesellschaftliche Determinanten produktiven } \\
\text { Alterns in Europa }\end{array}$ & 07 \\
\hline $153-07$ & $\begin{array}{l}\text { Axel Börsch-Supan } \\
\text { Christina B. Wilke }\end{array}$ & $\begin{array}{l}\text { Szenarien zur mittel- und langfristigen } \\
\text { Entwicklung der Anzahl der Erwerbspersonen } \\
\text { und der Erwerbstätigen in Deutschland }\end{array}$ & 07 \\
\hline
\end{tabular}

